\title{
Automatic Detection of Small Icebergs in Fast Ice Using Satellite Wide-Swath SAR Images
}

\author{
Ingri Halland Soldal ${ }^{1,2, *}$, Wolfgang Dierking $2,3\left(\mathbb{C}\right.$, Anton Korosov ${ }^{1}\left(\mathbb{D}\right.$ and Armando Marino ${ }^{4}(\mathbb{D})$ \\ 1 Nansen Environmental and Remote Sensing Center, Thormøhlens Gate 47, N-5006 Bergen, Norway; \\ anton.korosov@nersc.no \\ 2 Center for Integrated Remote Sensing and Forecasting for Arctic Operations, Department of Physics and \\ Technology, UiT-The Arctic University of Norway, 9019 Tromsø, Norway; wolfgang.dierking@awi.de \\ 3 Alfred Wegener Institute, Helmholtz Center for Polar and Marine Research, Bussestr. 24, \\ 27570 Bremerhaven, Germany \\ 4 Department of Biological and Environmental Sciences, University of Stirling, Stirling FK9 4LA, UK; \\ armando.marino@stir.ac.uk \\ * Correspondence: ingri.soldal@nersc.no
}

Received: 14 March 2019; Accepted: 31 March 2019; Published: 3 April 2019

\begin{abstract}
Automatic detection of icebergs in satellite images is regarded a useful tool to provide information necessary for safety in Arctic shipping or operations over large ocean areas in near-real time. In this work, we investigated the feasibility of automatic iceberg detection in Sentinel-1 Extra Wide Swath (EWS) SAR images which follow the preferred image mode in operational ice charting. As test region, we selected the Barents Sea where the size of many icebergs is on the order of the spatial resolution of the EWS-mode. We tested a new approach for a detection scheme. It is based on a combination of a filter for enhancing the contrast between icebergs and background, subsequent blob detection, and final application of a Constant False Alarm Rate (CFAR) algorithm. The filter relies mainly on the HV-polarized intensity which often reveals a larger difference between icebergs and sea ice or open water. The blob detector identifies locations of potential icebergs and thus shortens computation time. The final detection is performed on the identified blobs using the CFAR algorithm. About 2000 icebergs captured in fast ice were visually identified in Sentinel-2 Multi Spectral Imager (MSI) data and exploited for an assessment of the detection scheme performance using confusion matrices. For our performance tests, we used four Sentinel-1 EWS images. For judging the effect of spatial resolution, we carried out an additional test with one Sentinel-1 Interferometric Wide Swath (IWS) mode image. Our results show that only 8-22 percent of the icebergs could be detected in the EWS images, and over 90 percent of all detections were false alarms. In IWS mode, the number of correctly identified icebergs increased to 38 percent. However, we obtained a larger number of false alarms in the IWS image than in the corresponding EWS image. We identified two problems for iceberg detection: 1) with the given frequency-polarization combination, not all icebergs are strong scatterers at HV-polarization, and (2) icebergs and deformation structures present on fast ice can often not be distinguished since both may reveal equally strong responses at HV-polarization.
\end{abstract}

Keywords: iceberg detection; CFAR; iDPolRAD; SAR; optical images

\section{Introduction}

Icebergs present serious hazards for ship navigation and offshore installations. Consequently, there is a large interest to localize them timely and over vast areas. Because of their independence of cloud cover and daylight, satellite Synthetic Aperture Radar (SAR) images are among the preferred data sources for operational ice centers that are responsible for providing information on sea ice 
conditions and iceberg occurrences. The image spatial resolution mostly used for iceberg monitoring varies between a few and $100 \mathrm{~m}$. Processed SAR data are characterized by speckle noise, which causes a grainy appearance of the images making the identification of small icebergs extremely difficult. In particular, the following iceberg categories are affected: bergy bits (size 0-5 m), growlers (5-15 $\mathrm{m})$, and small bergs $(15-60 \mathrm{~m}$ ) (for iceberg categories see [1]). In many previous studies related to automatic detection of icebergs in SAR images reliable results were only obtained when focusing on icebergs in open water that cover several image pixels (e.g., [2-6]).

The Barents Sea represents the region where iceberg monitoring is especially needed because of various human activities including fishing, cargo shipping, and oil and gas exploration [7,8]. Marine terminating glaciers on Svalbard, Franz Josef Land (FJL) (see Figure 1), and Novaya Zemlya are the major sources of icebergs in the Barents Sea with typical iceberg sizes of $91 \mathrm{~m} \pm 53 \mathrm{~m} \times 64 \mathrm{~m} \pm$ $37 \mathrm{~m} \times 15 \mathrm{~m} \pm 7 \mathrm{~m}$ (length $\times$ width $\times$ height) [9]. The potential of identifying small icebergs in the Barents Sea in Radarsat ScanSAR and Envisat ASAR WideSwath and Alternate Polarization (HH, VV) imagery was investigated by Sandven et al. [10]. They compared SAR data to optical scenes from Landsat ETM+ and Terra ASTER on the test site in FJL and identified 15 icebergs of sizes between $50 \mathrm{~m}$ and $400 \mathrm{~m}$, trapped in fast ice. Their study was focused on the contrast between the single-polarization radar backscattering coefficients of icebergs and fast ice, considering differences in spatial resolution and incidence angle.

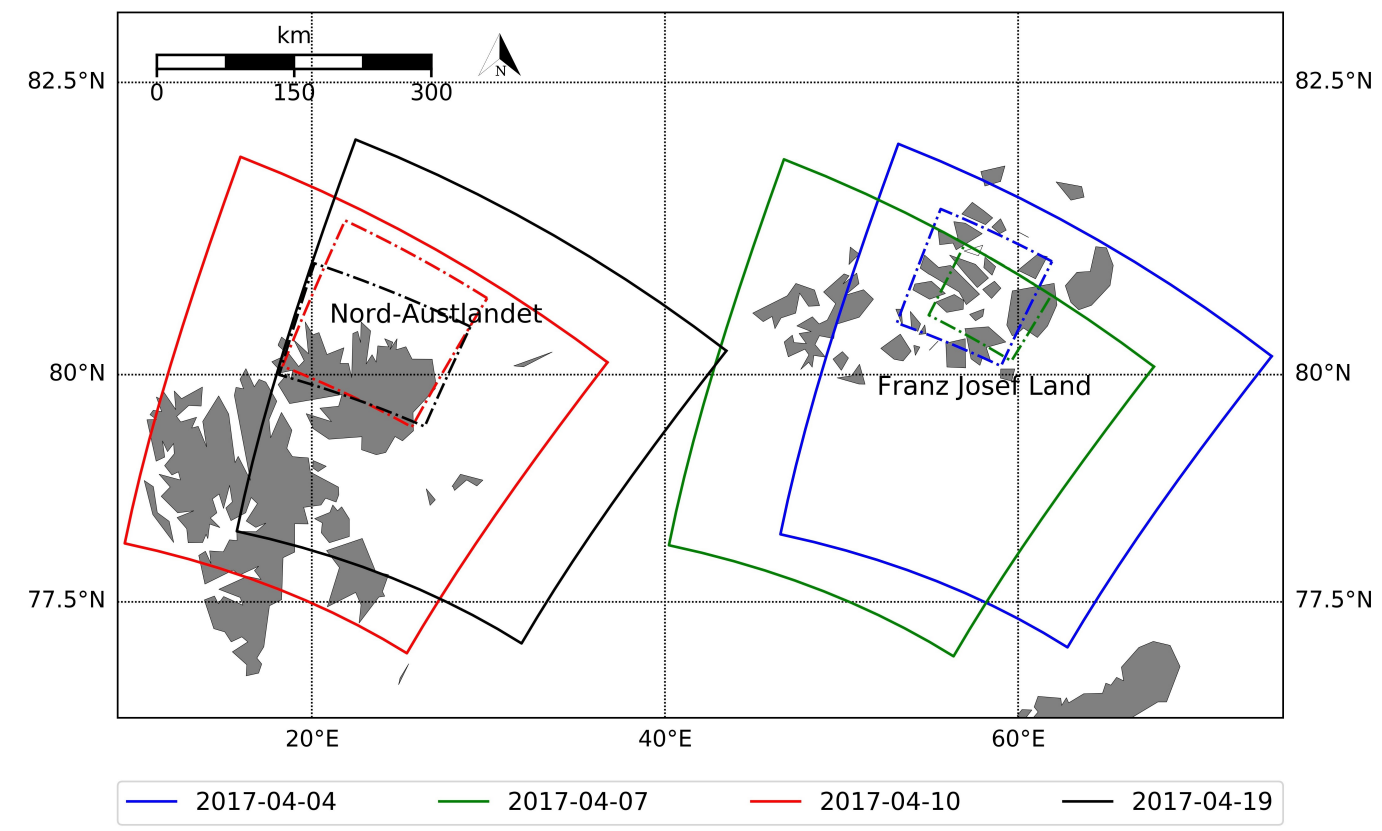

Figure 1. The Sentinel-1 image acquisitions used for automatic iceberg detection. The subimages with hatched outline are the regions used for testing and their colors match the corresponding acquisition.

Given the high importance of iceberg monitoring in the Barents Sea and the aforementioned challenges in their identification, we focus our study on the detection of very small icebergs in particular in the region of FJL and the northern side of Nord-Austlandet (NA), Svalbard (Figure 1). The goal is to test a new method based on dual-polarization SAR images and to quantify the limitations of the coarse-resolution SAR imagery typically used in operational ice charting.

We deal with icebergs trapped in land fast ice, defined as sea ice that is attached to land and stays at the same place over a long time due to little impact by currents and winds [11]. From the methodological point of view, land fast ice is chosen because most of the icebergs there are stationary [10]. This makes it very easy to validate iceberg detections in SAR imagery using optical satellite images which were acquired with a time difference from hours to several days. On the other hand, the icebergs will eventually be released into the open ocean when the land fast ice melts and 
may cause a threat for operations in close vicinity. It is, therefore, necessary to be able to detect these icebergs and include them in a safety analysis. In FJL the fjords are covered by land fast ice until the end of April, and the glaciers calve many icebergs into the fast ice. At NA there is also a large region of land fast ice, but due to the impact of the drifting sea ice pushing against it, the fast ice surface is rough, which complicates the detection of icebergs. The density of icebergs observed at NA is also lower than what is observed in the FJL region. Similar results can be expected for drifting sea ice with comparable surface characteristics (cm- to - m-scale roughness) and volume properties (salinity, air bubble inclusions). However, regions of drifting ice with low areal concentrations (e.g., at the ice margin) represent a more challenging situation since open water or brash ice between the consolidated ice floes cause more complex backscattering patterns in the radar image.

In our study, we take direct advantage of the dual-polarization mode offered by the Sentinel-1 EWS mode. The proposed detection scheme is based on the work by Marino et al. [12] who developed a method that uses both HH- and HV- (or VV- and VH-) polarized SAR data to significantly increase the contrast between icebergs and their background (which in general can be drifting sea ice, fast ice, and open water). This method is called the intensity Dual-Polarization Ratio Anomaly Detector (iDPolRAD). As identifications of icebergs smaller than about $100 \mathrm{~m}$ are more reliable in optical than in SAR images [10], we further extend the previous studies by visual identification of more than 6000 reference icebergs on Sentinel-2 imagery (of which we finally used about 2500 for investigating the results of automatic detection). In the fast ice and under favourable light and cloud conditions, the icebergs stand out because of a dark shadow due to the low sun angle and the iceberg freeboard above the fast ice surface. The manual identification of such a large number of icebergs in high-resolution optical images (10 $\mathrm{m}$ for Sentinel-2 visible bands) for validating an algorithm for iceberg detection in SAR images is unique to our study.

Using the iDPolRAD together with a modified CFAR detector we are here assessing the feasibility of automatic detection of icebergs as small as a single image pixel. The CFAR performance depends on the choice of the probability density function (PDF) of the background clutter and on the threshold for detection (see e.g., [13,14]). We applied the Gamma-, Generalized Gamma-, and Generalized Extreme Value distributions to select a PDF that best matches the iDPolRAD filtered values.

The paper starts with a description of the SAR data and the collection of validation data (Section 2). Subsequently we go through the steps of our algorithm for iceberg detection (Section 3), and present the results for our test sites (Section 4). The paper proceeds with a discussion concerning scattering characteristics and incidence angle sensitivities of icebergs (Section 5) and ends with the conclusions (Section 6).

\section{Data}

The Sentinel-1 SAR is operating at C-band, providing several imaging modes that differ in swath width and spatial resolution [15]. For testing the iDPolRAD automatic detection of icebergs we use images acquired in EWS mode (Table 1), which are processed to ground range at medium resolution (Figure 1). The EWS mode provides a swath width of $410 \mathrm{~km}$ with a spatial resolution of $90 \mathrm{~m}$ and a pixel size of $40 \mathrm{~m}$. The wide swath makes this mode preferable for operational monitoring as the Barents Sea can be covered up to twice a day. The Noise-Equivalent-Sigma-Zero (NESZ) lies between $-23 \mathrm{~dB}$ and $-34 \mathrm{~dB}$, dependent on the incidence angle and the position in the different beams of which the EWS image is combined, see [16]. The equivalent number of looks, ENL, which is a measure for the influence of speckle, is 10.7. We also carried out tests with the IWS high resolution mode, for which the following numbers are valid: spatial resolution $20 \mathrm{~m}$, pixel size $10 \mathrm{~m}$, swath width $250 \mathrm{~km}$, NESZ between $-24 \mathrm{~dB}$ and $-30 \mathrm{~dB}$, and ENL $=4.4$ [16]. The Sentinel-1 data were radiometrically calibrated using the Nansat software [17]. The normalized radar backscatter cross-section $\left(\sigma^{0}\right)$ was calculated from the TIFF file using the calibration lookup table provided in the accompanying metadata XML [18]. The pixels that belong to land [19] and glaciers were discarded from the analysis. The landmask has a resolution of $250 \mathrm{~m}$. The absolute location accuracy of the medium-resolution EWS product is not 
specified in the User's Manual. For the IWS mode it is $7 \mathrm{~m}$. Korosov and Rampal [20] found that the accuracy of georeferencing was about 5 pixels (i.e., $200 \mathrm{~m}$ ) for the EWS medium resolution product.

Sentinel-2 carries the MSI that samples 13 bands in the range from $440 \mathrm{~nm}$ to $2200 \mathrm{~nm}$ at a swath width of $290 \mathrm{~km}$. We used bands 2, 3, and 4 located in the visible range with a spatial resolution of $10 \mathrm{~m}$. The images from Sentinel-2 were combined with the SAR data using Google Earth Engine (GEE, [21], see Figure 2). Due to darkness and bad weather conditions during image acquisitions, data from only a few days were available for the test regions FJL and NA. Over 6000 icebergs were manually detected and measured by identifying brighter spots combined with shadows (Figures 2 and 3, Table 2), recording the iceberg coordinates, and drawing polygons around the identified icebergs.

Table 1. Date, time and region of each Sentinel-1 EW image acquisition, including the name of the respective files. The last two columns give the size and incidence angle range of the part of image included in the automatic detection.

\begin{tabular}{|c|c|c|c|c|}
\hline $\begin{array}{l}\text { Date (Year-Month-Day), } \\
\text { Time }\end{array}$ & Region & S1-File & $\begin{array}{c}\text { Part of Image } \\
\text { [No of Rows, } \\
\text { No of Columns] }\end{array}$ & $\begin{array}{c}\text { Incidence Angle } \\
\text { Range }\left[{ }^{\circ}\right]\end{array}$ \\
\hline $\begin{array}{c}\text { 2017-04-04 } \\
03: 38: 14\end{array}$ & FJL & $\begin{array}{l}\text { S1A_EW_GRDM_1SDH_20170404T033814_- } \\
\text { 20170404T033918_015989_01A5F5_FB23 }\end{array}$ & $3000 \times 3000$ & $36-43$ \\
\hline $\begin{array}{l}2017-04-07 \\
04: 02: 57\end{array}$ & FJL & $\begin{array}{l}\text { S1A_EW_GRDM_1SDH_20170407T040257_ } \\
\text { 20170407T040402_016033_01A74D_1347 }\end{array}$ & $2500 \times 2000$ & $28-35$ \\
\hline $\begin{array}{l}2017-04-10 \\
06: 06: 23\end{array}$ & NA & $\begin{array}{c}\text { S1A_EW_GRDM_1SDH_20170410T060623_ } \\
\text { 20170410T060727_016078_01A8B3_8486 }\end{array}$ & $4000 \times 4000$ & $30-40$ \\
\hline $\begin{array}{c}2017-04-19 \\
05: 41: 40\end{array}$ & NA & $\begin{array}{c}\text { S1A_EW_GRDM_1SDH_20170419T054140_ } \\
\text { 20170419T054244_016209_01ACB5_82A4 }\end{array}$ & $3000 \times 4300$ & $36-46$ \\
\hline
\end{tabular}

Table 2. Date, time and region of Sentinel-2 MSI image acquisitions and number of manually detected icebergs. The rightmost column give the air temperature at 12:00 UTC at the day of the acquisition. The temperature data measured at $2 \mathrm{~m}$ (NA) and $22 \mathrm{~m}$ (FJL) was retrieved from data pools of the Norwegian Meteorological Institute and the University of Wyoming, respectively.

\begin{tabular}{ccccc}
\hline $\begin{array}{c}\text { Date (Year-Month-Day), } \\
\text { Time }\end{array}$ & Region & $\begin{array}{c}\text { Ice Type } \\
\text { [Fast Ice] }\end{array}$ & $\begin{array}{c}\text { Number of } \\
\text { Manually Detected } \\
\text { Icebergs }\end{array}$ & $\begin{array}{c}\text { T } \\
\mathbf{1 2 : 0 0 ~ U T C} \\
{\left[{ }^{\circ} \mathrm{C}\right]}\end{array}$ \\
\hline $\begin{array}{c}\text { 2017-04-04 } \\
\text { 11:46:41 }\end{array}$ & FJL & Smooth & 2292 & -20.7 \\
$\begin{array}{c}\text { 2017-04-07 } \\
\text { 11:56:40 }\end{array}$ & FJL & Smooth & 2940 & -19.9 \\
$\begin{array}{c}\text { 2017-04-10 } \\
13: 47: 25\end{array}$ & NA & Rough & 688 & -9.7 \\
$2017-04-19$ & & & & -12.5 \\
$14: 17: 39$ & NA & Rough & 827 & \\
\hline
\end{tabular}




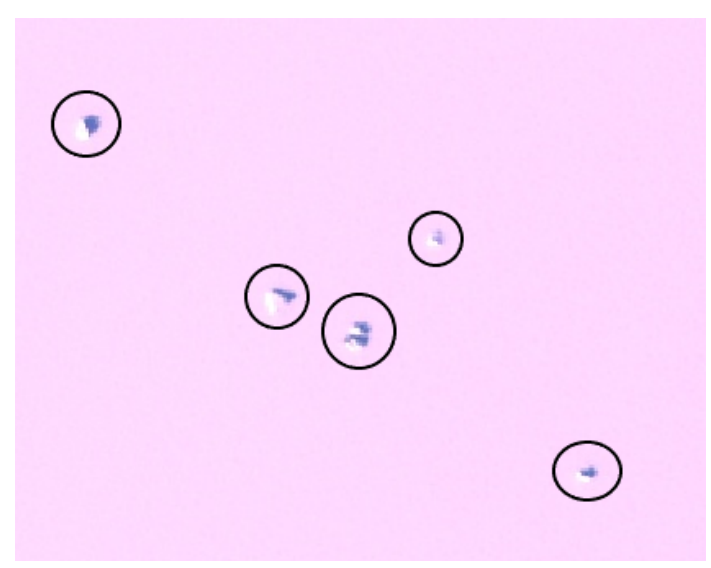

(a)

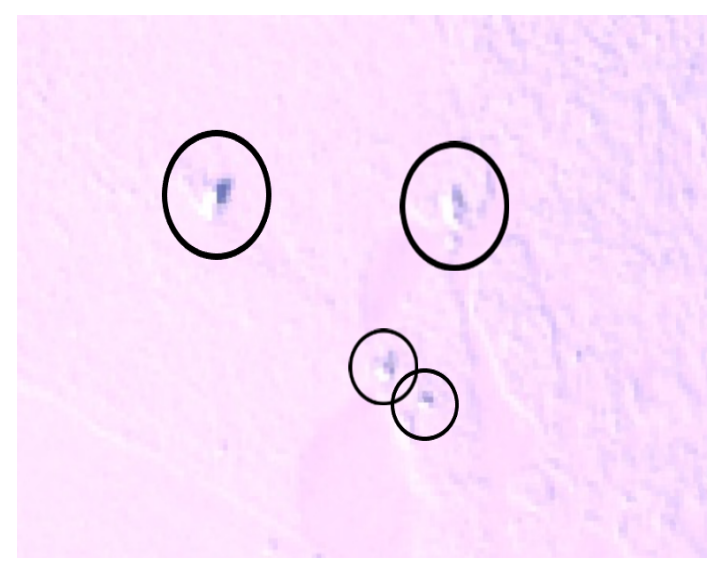

(b)

Figure 2. RGB composite from B4, B3, B2 bands of Sentinel-2 MSI showing manually detected icebergs in (a) smooth fast ice at Franz Josef Land on 4 April 2017 (image size 2800 by $1950 \mathrm{~m}$ ) and (b) rough fast ice at Nord-Austlandet, Svalbard, on 10 April 2017 (image size 1100 by 800 m).

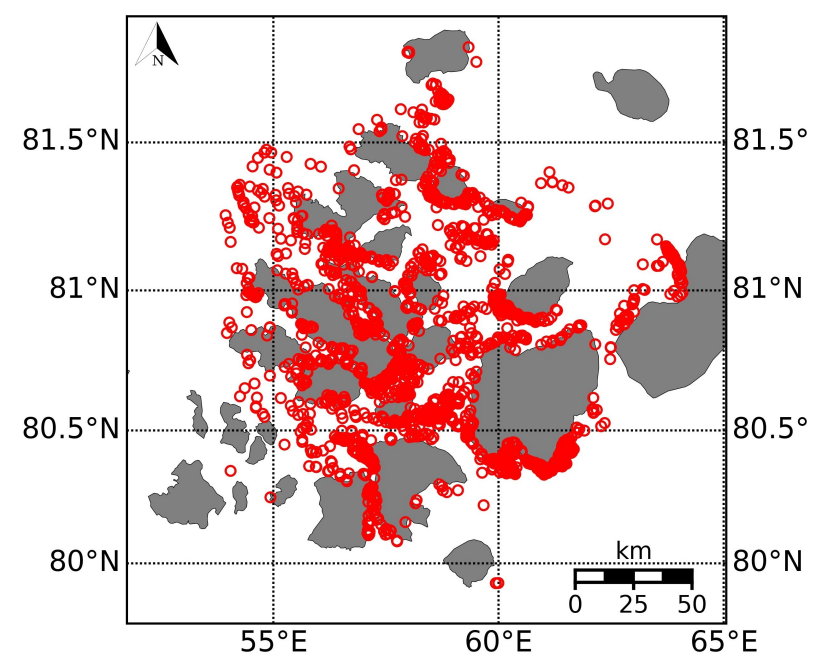

Figure 3. A total of 2292 icebergs manually detected in Sentinel-2 image over Franz Josef Land on 4 April 2017. Grey areas are land. Iceberg positions located in the landmask may be due to geolocation errors or details such as bays that are not present in the landmask.

\section{Methods}

\subsection{Overview}

In the following, we present the algorithm for automatic iceberg detection and the method for investigating its performance (Figure 4). We start by introducing the iDPolRAD filter that is used to increase the contrast between icebergs and the surrounding fast ice (Section 3.2). Then we describe a blob detector which we apply in a pre-processing step to select those pixels that reveal a higher value of the iDPolRAD relative to the surrounding pixels, hence indicating a larger probability of representing an iceberg (Section 3.3). The selected pixels are input for the next step, which is a modified CFAR detector (Section 3.4). Finally, we compare the output of the automatic detection to the manually identified icebergs, introducing the corresponding confusion matrix in Section 3.4.4. 


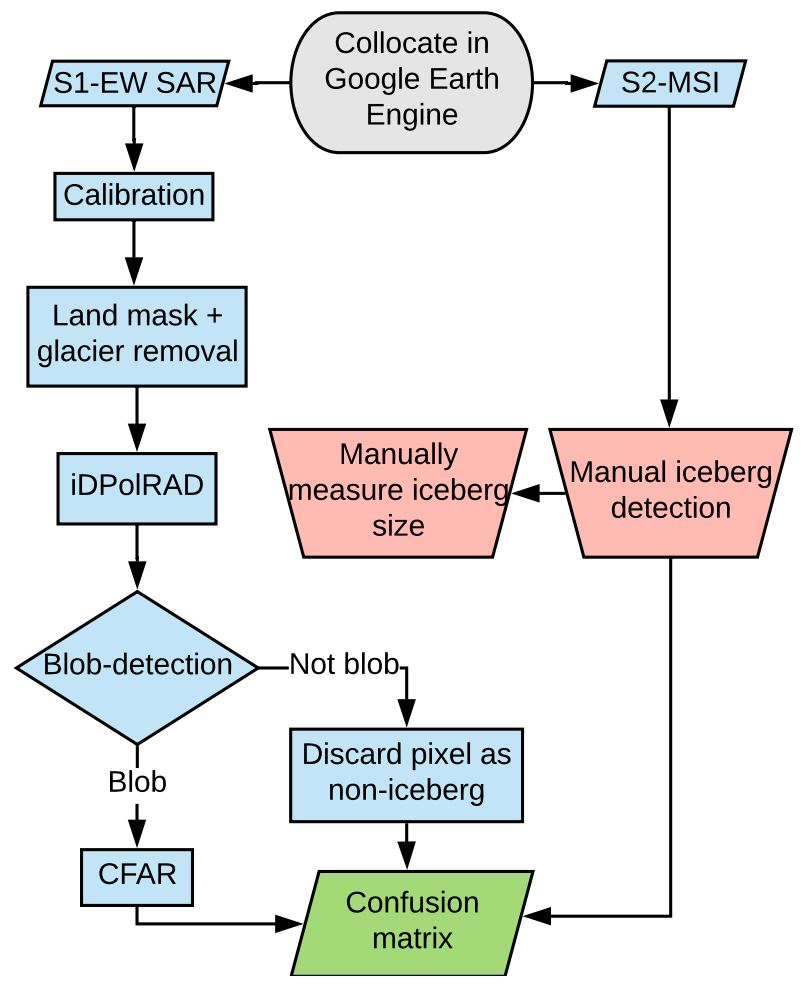

Figure 4. Schematic overview of the algorithm for automatic iceberg detection using Sentinel-1 SAR data, and the validation of the algorithm using manual detections from Sentinel-2 MSI.

\section{2. iDPolRAD}

A filter to increase the contrast between icebergs and the surrounding sea ice or open water was proposed by Marino et al. [12]. It is called the iDPolRAD and was designed and tested using dual-polarized incoherent Sentinel-1 data [12]. The filter is based on the observation that icebergs often exhibit a different polarimetric behaviour compared to sea ice and open water [22-24]. This is in particular valid for icebergs which represent a volume of freshwater ice. Under dry, freezing conditions they can be penetrated by radar waves, hence volume scattering contributes significantly to the observed radar signatures. The surfaces of icebergs are often characterized by the presence of highly reflective planes with random orientations which dominate the scattering if the icebergs are wet or toppled-over. Scattering from the ocean, and often from saline sea ice is determined by surface properties (C-band radar: roughness on cm-scale, dielectric constant). Therefore, wet and turned-over icebergs may reveal similar scattering characteristics as the surrounding water or sea ice. If volume scattering is significant and if the orientations of larger reflective planes (a few decimeters to meters in size) are random, icebergs are expected to have a large cross-polarization contribution and a large depolarization ratio (defined as the ratio between the intensity of cross- over co-polarization channels). In the original algorithm, two boxcar filters are applied over the HV and HH intensity images, exploiting two different window sizes: a smaller test window, $W_{\text {test }}$, located in the center of a larger training window, $W_{\text {train }}$. The detector can be written as:

$$
\Lambda=\frac{<|H V|^{2}>_{\text {test }}-<|H V|^{2}>_{\text {train }}}{<|H H|^{2}>_{\text {train }}}>T_{\Lambda}
$$

where $<>_{\text {test }}$ and $<>_{\text {train }}$ identify the spatial average using the test and training windows, respectively, and $T_{\Lambda}$ is a threshold.

This detector is scale invariant (built as a ratio between intensities), however scale invariance may be disadvantageous if we want to detect bright targets. To regain information about the intensity we 
can multiply the ratio by an intensity image. In this context, the cross-polarization channel is preferred because it shows a higher contrast between icebergs and clutter:

$$
I=\Lambda \cdot<|H V|^{2}>_{\text {test }}
$$

If a pixel in the test window presents an increase in volume scattering or oriented reflections relative to the background in the training window, $\Lambda$ reveals a large positive value. For a homogeneous area, test and training window reveal no difference, hence $\Lambda$ is zero. The iDPolRAD can also assume negative values, e.g., if the test window covers an open water patch in sea ice which is characterized by a very low $\mathrm{HV}$-polarization response while the training window is dominated by the surrounding rough multi-year ice revealing a larger HV-intensity level.

The combination of test and training window is moved pixel by pixel along lines and columns of the image. Any bright object (of one to a few pixels in size) located in the training window affects the output of the iDPolRAD filter (the contamination effect). We did not remove bright objects at this stage of processing, since the application of the iDPolRAD filter still results in contrast enhancement even in the presence of bright objects in the training window. We observed a margin around the object under test where the output of the iDPolRAD filter was much larger than the background level. By using Gaussian weights instead of a boxcar function for the training window this effect was avoided.

\subsection{Blob-Detector}

To apply the CFAR detector (including determination of the background statistics) on every pixel in an image can be a very time consuming task. To speed up the algorithm we therefore first apply a blob detector to select only pixels with a higher probability of being icebergs before carrying out the final detection. The blob detection is based on the scikit-image library in Python [25] as explained below.

A blob consists of one or more pixels that reveal an intensity contrast with respect to the background (in the extreme case black on white or white on black). Blob detection is performed in the following steps [25]. First, the input SAR image is processed with a set of Gaussian filters with different values of standard deviation $S$ (i.e., radius of the filter), calculated in sliding windows. The next step is to apply a Laplacian filter to obtain maximum and minimum values, and multiply with the corresponding $S^{2}$ to provide spatial scale invariance. That operation produces a N-layer "cube" in scale-space volume representing $\mathrm{N}$ spatial scales. The pixels that represent a local maximum (above a given threshold) with respect to their 26 neighbours (in three dimensions) are selected as blobs [26].

There are two important parameters for this detector; the minimum threshold for blob-detection $\left(\tau_{B}\right)$ and the maximum standard deviation of the Gaussian filter, $S_{M}$. The threshold $\tau_{B}$ is the lowest value a pixel can have to be considered a blob, while the value of $S_{M}$ is important to make sure the detector includes the largest features. The iDPolRAD value of the iceberg pixels varies significantly. In order to find the optimal choice for $\tau_{B}$ we have performed blob detection with several thresholds and validated with manually detected icebergs. For $S_{M}$ it is important not to choose a too large value for the blob detection, but rather to base it on the known "typical" size distribution of the icebergs. If $S_{M}$, and thus the radius of the blob, becomes too large, smaller icebergs that are close to each other may be considered as one blob instead of two (or more). We note that the Laplacian of Gaussian blob detector can only handle bright objects on a darker background.

Due to slight differences in the geolocations of data in GEE and the downloaded Sentinel-1 SAR images, some manual detections are not correctly localized at the position of peaks in the SAR image. This is solved by automatic shifting of the manually detected icebergs into the nearest blob if it exists within a given distance $(200 \mathrm{~m} / 5$ pixels). The blobs that are coincident with manually detected icebergs within this distance are true positive detections, while the blobs that are not coinciding with manually detected icebergs are denoted as false positive detections (see notations in Table 3). 
Table 3. The names of the detected objects used hereafter.

\begin{tabular}{ll}
\hline Name & Description \\
\hline True Positives $\left(T P_{B}\right)$ & $\begin{array}{l}\text { Pixels that are selected by automatic blob detection } \\
\text { and are manually defined as icebergs }\end{array}$ \\
\hline False Positives $\left(F P_{B}\right)$ & $\begin{array}{l}\text { Pixels that are selected by automatic blob detection } \\
\text { but are not manually defined as icebergs }\end{array}$ \\
\hline False Negatives $\left(F N_{B}\right)$ & $\begin{array}{l}\text { Pixels that are not selected by automatic blob detection } \\
\text { but are manually defined as icebergs }\end{array}$ \\
\hline True Negatives $\left(T N_{B}\right)$ & $\begin{array}{l}\text { Pixels that are not selected by automatic blob detection } \\
\text { and are not manually defined as icebergs }\end{array}$ \\
\hline
\end{tabular}

\subsection{A Modified Constant False Alarm Rate Algorithm}

Since we carry out the iceberg detection on iDPolRAD instead of intensity values we fitted different PDFs to the distributions of the iDPolRAD-values (Sections 3.4.1 and 3.4.2) and evaluated the quality and robustness of the respective matches (Section 3.4.3). Finally, we tuned the parameters of the CFAR algorithm using the manually detected icebergs (Section 3.4.4).

\subsubsection{The Modified CFAR Approach}

The basic CFAR approach is as follows: A pixel is marked as an iceberg if the SAR signal intensity $\left(\sigma^{0}\right)$ exceeds a threshold (T). This threshold is chosen such that the integral of the tail of the SAR signal intensity PDF $\left(f\left(\sigma^{0}\right)\right)$ is equal to or smaller than a critical Probability of False Alarm (PFA) (Figure 5). The PDF is obtained from the histogram of the intensity values in a training window surrounding a smaller window in the center (e.g., [14]). In our modified CFAR we determine the background PDF from the iDPolRAD values, denoted as I: from the histogram H(I), obtained from a training window surrounding the pixel under test, we consider only image pixels between an upper and a lower boundary. The lower value is denoted $I_{\min }$ and represents the peak of the probability density function, $P D F_{\max }$. The upper value is $I_{\max }$ and corresponds to a preselected percentile $P_{2}$ relative to all values within each training window. The motivation for this procedure is to: (a) exclude impacts of noise at the lower end of the histogram and the influence of pixels representing icebergs (with typically high iDPolRAD-values) at its higher end, and (b) increase the robustness of fitting the PDF to $\mathrm{H}(\mathrm{I})$ by excluding the steep leading edge of the histogram. This procedure is suitable because for calculations of the PFA we are mainly interested in a close match of the tail region representing only the background. In our analysis we found that the histograms of I increase and decrease very sharply around their peaks. This makes it very difficult to find a theoretical PDF that matches the shape of the histogram sufficiently well over the full range of iDPolRAD values. The value for $I_{\min }$ is found by smoothing the histogram successively using a running average over three bins and retrieving the iDPolRAD-value at the index of the maximum of the smoothed histogram. The CFAR detector uses a sliding window of size $W_{C F A R}$, checking whether the center pixel of the window is an iceberg or not. Thus, the background is defined relative to each pixel under test. To reduce the computation time we applied the CFAR detector on blob-detected pixels only. 


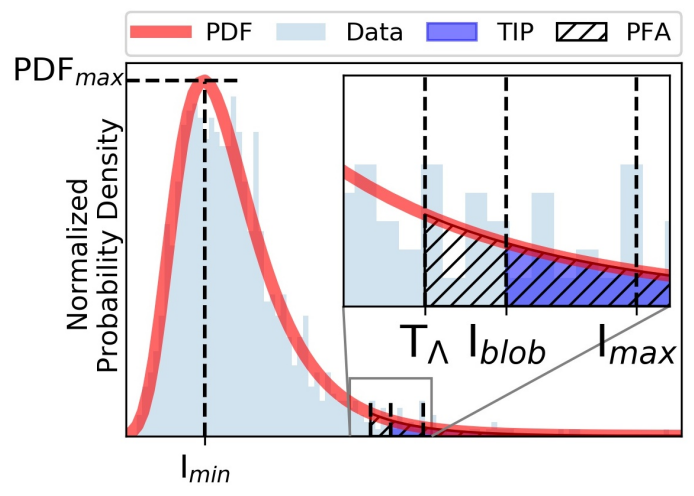

Figure 5. Schematic representation of PDF fitting to the histogram of the data. The dark blue shaded area represents the TIP (see Section 3.4.4) and the hatched region represents the area corresponding to the PFA. $T_{\Lambda}$ is the threshold, $I_{\min }$ and $I_{\max }$ are the lower and upper boundaries used to represent the background data respectively, and $I_{b l o b}$ is the value of the blob-detected pixel.

\subsubsection{Fitting of the Probability Density Functions}

Since we analyse iDPolRAD-values, I, instead of intensities, $\sigma^{0}$, we had to consider that the PDFs over I are not necessarily the same as typically used for $\sigma^{0}$. Three PDFs, selected based on the observed histogram shapes, have been tested for representing the background data in the image: Gamma-, Generalized Gamma-, and the Generalized Extreme Value (GEV) distributions. The PDFs are presented in Table 4. The scipy.stats module in Python [27] was used to obtain the parameters and final distributions for the PDFs.

Table 4. Equations and symbols for the different PDFs. I is here the iDPolRAD values.

\begin{tabular}{llr}
\hline PDF & Equation & Parameters \\
\hline Gamma & $f(I, a)=\lambda^{a} I^{a-1} \frac{e^{-\lambda I}}{\Gamma(a)}$ & $\begin{array}{r}\text { a = shape parameter } \\
\lambda=\text { inverse scale parameter }\end{array}$ \\
\hline Generalized Gamma & $f(I, a, c)=|c| I^{c a-1} \frac{e^{-I c}}{\Gamma(a)}$ & a, c = shape parameter \\
\hline Generalized Extreme Value & $f(I, c)=e^{-e^{-I}} e^{-I}$, if $\mathrm{c}=0$ & $\mathrm{c}=$ shape parameter \\
& $f(I, c)=e^{-(1-c I)^{\frac{1}{c}}}(1-c I)^{\frac{1}{c}-1}$ & \\
\hline
\end{tabular}

\subsubsection{Quality and Robustness of Probability Density Functions}

To check the representativeness of the PDF fit, the PDF parameters and corresponding distributions were calculated both for a larger area within one of the SAR images from FJL (size $3000 \times 3000$ pixels) as well as for approximately 9700 windows of $141 \times 141$ pixels within the image, using blob-detected pixels as central window point. Pixels within the window with values below or above $I_{\min }$ and $I_{\max }$ were excluded from the calculations (hence alleviating or even avoiding contamination, see above). The resulting PDFs and their parameters were compared to the corresponding histogram to assess the quality of the fit. To this end the mean absolute error between the histogram and the corresponding PDF, multiplied by the bin width, was determined. This procedure is similar to the Kolmogorov-Smirnov test [28]. The statistics for the smaller windows were also compared to the statistics of the large area to get a measure of the variability of the PDFs with respect to the data. Because the windows are located in different parts of a SAR image we cover different incidence angles (see below). 


\subsubsection{Determination of Thresholds for the Modified CFAR}

The type of PDF and the PFA value are crucial to the CFAR algorithm for iceberg detection. True and false positives from blob detection were used for determination of the threshold to distinguish icebergs and background (note that icebergs identified in the Sentinel-2 image that do not appear in the SAR image - the false negatives—can not be accounted for in the PFA).

For each selected object (iceberg, i.e., true positive, or "non-iceberg", i.e., false positive) the PDF parameters were calculated using the surrounding background pixels within the CFAR window of size $141 \times 141$ pixels. Then, the corresponding PDF was calculated for each CFAR window and integrated between the iDPolRAD value of the object obtained from blob detection and infinity to obtain a value of 'Tail Integrated Probability (TIP)' (see Figure 5 and Equation (3)).

$$
T I P=\int_{I[b l o b]}^{\infty} f(x) d x
$$

TIP is small for objects with high intensity and low values of $\mathrm{f}(\mathrm{x}), \times>I_{\text {blob }}$ (typical for icebergs characterized by strong scattering). From the resulting TIP values the PFA can be selected to optimally distinguish between icebergs and background excluding as many non-icebergs as possible. To this end we prepared scatter plots that present the TIP values for true and false positives. In comparison to the manually identified icebergs we obtain four groups of objects (notation see Table 3 in Section 3.3, replacing " $\mathrm{B}$ " with "CFAR" for iceberg detection) that allow to assess the quality of the algorithm using the confusion matrix (see Table 5).

Table 5. Confusion matrix for assessing quality of automatic iceberg detector.

\begin{tabular}{lll}
\hline Total $=T P_{C F A R}+F P_{C F A R}+F N_{C F A R}+T N_{C F A R}$ & Actual Iceberg & Actual Non-Iceberg \\
\hline Predicted iceberg & $T P_{C F A R}$ & $F P_{C F A R}$ \\
Predicted non-iceberg & $F N_{C F A R}$ & $T N_{C F A R}$ \\
\hline
\end{tabular}

\section{Results}

\subsection{Detection Performance of the iDPolRAD-Filter}

In the following example we demonstrate the effect of the iDPolRAD filter. We used a single pixel as test window, and a training window weighted with a two-dimensional Gaussian function with a standard deviation $\sigma$ equal to 7 . In the implementation this corresponds to a window size of $57 \times 57$ pixels, where the maximum of the filter is located over the center and decreases to zero at the margin. By comparing the iDPolRAD output using a range of values for $\sigma$ and test windows of different sizes, we found that the chosen values gave the best result. A test window that covers only one pixel (corresponding to a size of $40 \times 40 \mathrm{~m}$ ) is optimal since many icebergs in the Barents Sea are even smaller than this size. In the case that they are larger, this is considered in the final step of detection where neighbouring detected pixels are merged. The result of the iDPolRAD filter is presented in Figures 6 and 7. The figures show that the background clutter is highly reduced while the bright features, such as icebergs, are enhanced when we apply the iDPolRAD filter. This increased contrast can be exploited in the CFAR detector. In an additional test we studied the relationship between the iDPolRAD value and the size of icebergs and found that no correlation exists between both parameters. 


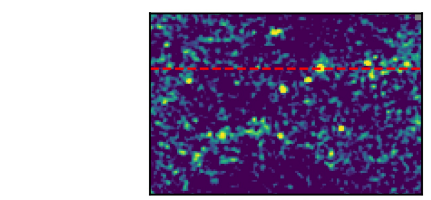

(a) $[\mathrm{dB}]$
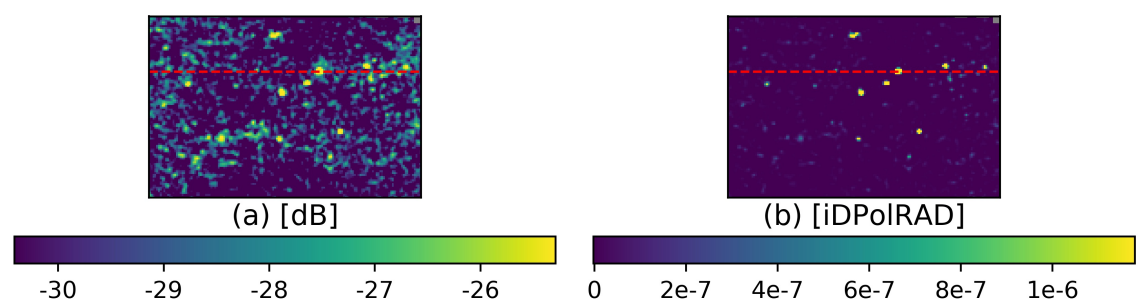

Figure 6. (a) $\sigma_{H V}^{0}$, (b) iDPolRAD filter. The scene is from Franz Josef Land on 4 April 2017, showing icebergs in frozen sea ice. The contrast between background intensity variations and icebergs is strongly enhanced after applying the iDPolRAD filter. The red hatched line shows the transect presented in Figure 7 .

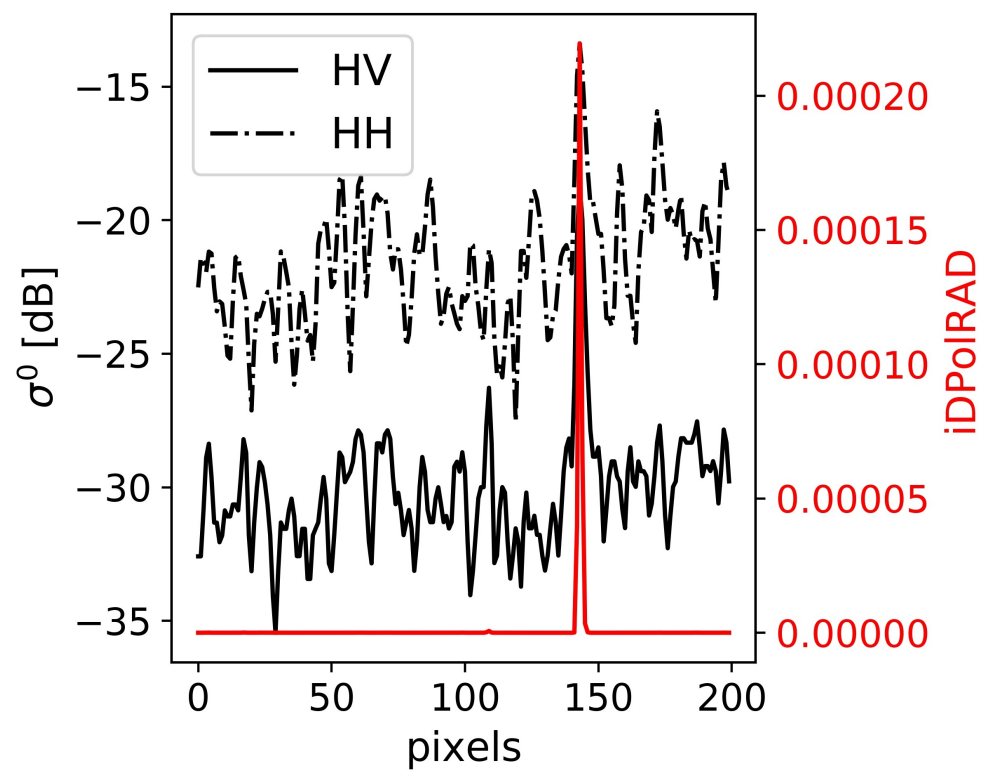

Figure 7. A transect from Figure 6 showing how the iDPolRAD filter enhances the difference between iceberg-value and background-values compared to $\mathrm{HH}$ and $\mathrm{HV}$ intensities.

\subsection{Blob Detection Results}

The numbers of $T P_{B}, F N_{B}$ and $F P_{B}$ from different thresholds of blob detection are presented in Tables 6 and 7. In general a lower $\tau_{B}$ will detect most of the blobs found for the higher value of $\tau_{B}$, but in addition many additional ones (Table 6). For $\tau_{B}=1 \times 10^{-7}$ the number of $T P_{B}$ was high: 677 icebergs were detected, only $187\left(F N_{B}\right)$ were missed. However, the number of $F P_{B}$ was extremely large. By choosing $\tau_{B}=1 \times 10^{-6}$ the value of $F P_{B}$ was reduced by a factor of almost 6 , but more than 50 percent of the icebergs were not detected $\left(T P_{B}=321, F N_{B}=489\right)$. Therefore, we choose to continue with an intermediate threshold $\left(\tau_{B}=5 \times 10^{-7}\right)$ in the following processing steps. Many icebergs reveal an iDPolRAD value lower than $\tau_{B}$ but an acceptable balance between true detections and false alarms makes it unfavourable to use too low values.

By choosing $S_{M}=0.1$ we detect blobs that have a radius of 0.9 pixels, and thus a diameter close to 2 pixels, or larger. The minimum iceberg diameter observed in the optical image was approximately $10 \mathrm{~m}$, and we could therefore have chosen a value of $S_{M}$ that was even lower. However, with a smaller blob the computation is more time-consuming and the number of false alarms increases. We therefore refrained from using a smaller radius. The computation time needed for blob detection with our choice of input parameters is $36 \mathrm{~min}$ which is too long for operational applications. In this study, however, we did not attempt to optimize computation time but focused entirely on the sensitivity of detection results when changing the required input parameters. 
In Table 7 , results for $T P_{B}, F N_{B}$ and $F P_{B}$ are shown for different values of $S_{M}$. For $S_{M}=0.1$ and $S_{M}=0.5$ (radius of 0.9 and 2.5 pixels, respectively), only one layer was used, i.e., maxima were determined only in one spatial scale. In case of larger radii, two layers were combined, one with $S_{M}=2$ and the second with $S_{M}=3$ (radius of 8.5 and 12.5 pixels, respectively), hence considering different spatial scales. The results in Table 7 indicate that larger icebergs were not present in our SAR scenes, hence the detection of maxima on different spatial scales is not necessary. We found that in general all blobs detected at a higher value of $S_{M}$ were also detected at lower $S_{M}$-values. However, in a few cases we observed that some blobs were only detected at larger values of $S_{M}$. This could be a result of the Laplacian of Gaussian if many high-value pixels are located close to one another. In this case the maxima in the high-resolution layer of the cube (smaller $S_{M}$ ) may not be unique with respect to neighbouring pixels and will therefore not be considered a blob for the smaller $S_{M}$.

In Figure $8 T P_{B}, F N_{B}$ and $F P_{B}$ are presented visually for $\tau_{B}=5 \times 10^{-7}$ and $S_{M}=0.1$ using an image from FJL. The results of blob detection for all images of test sites FJL (4 and 7 April) and NA (10 and 19 April) are listed in Table 8. They reveal a drastic increase of false positives in the rough fast ice at the NA test site. It has to be kept in mind that the blob detection is not equivalent to iceberg identification. The motivation for using blob detection is to reduce the computational effort by carrying out the final CFAR-based detection only on the blobs. Nevertheless it is interesting to note that true positives (i.e., icebergs) were already lost at this stage (see Section 5).

For the rough sea ice the blob detector responds not only to icebergs with strong scattering intensities but also to deformation structures of the sea ice cover which may reveal a similarly strong radar backscattering. As Table 8 shows, we obtained between almost 100,000 and 150,000 false alarms (with $S_{M}=0.1$ ). Blob detection as first step to iceberg identification can hence only be sufficiently successful if it is focused on larger icebergs (i.e., larger spatial scales). But even then, larger sea ice deformation structures may be confused with icebergs (see, e.g., [2]).

Note that the number of identified icebergs $\left(T P_{B}+F N_{B}\right)$ was much lower in Tables 6 and 7 than the number presented in Table 2. This is due to the fact that many icebergs were discarded after the images had been filtered with the landmask as they were too close to land and glaciers. The total number of icebergs can also be different between different values of $\tau_{B}$ due to the relocation of icebergs to the closest blob (see Section 3.3). This may change some iceberg pixels from having NaN (in the area of the landmask) to get a physical iDPolRAD-value while others will remain NaNs.

Table 6. $T P_{B}, F N_{B}$ and $F P_{B}$ with different $\tau_{B}$ for blob detection with $S_{M}=0.1$ in the Sentinel 1 image from 4 April 2017. The last column indicates the computation time in seconds.

\begin{tabular}{lllll}
\hline $\boldsymbol{\tau}_{\boldsymbol{B}}\left(\boldsymbol{S}_{\boldsymbol{M}}=\mathbf{0 . 1}\right)$ & $\boldsymbol{T P}_{\boldsymbol{B}}$ & $\boldsymbol{F N _ { \boldsymbol { B } }}$ & $\boldsymbol{F P}_{\boldsymbol{B}}$ & Time [s] \\
\hline $1 \times 10^{-6}$ & 321 & 489 & 14,492 & 533 \\
$5 \times 10^{-7}$ & 413 & 400 & 28,090 & 2172 \\
$1 \times 10^{-7}$ & 677 & 187 & 81,390 & 18,498 \\
\hline
\end{tabular}

Table 7. $T P_{B}, F N_{B}$ and $F P_{B}$ with different $S_{M}$ for blob detection with $\tau_{B}=5 \times 10^{-7}$ in the Sentinel 1 image from 4 April 2017. The last column indicates the computation time in seconds.

\begin{tabular}{lllll}
\hline $\boldsymbol{S}_{\boldsymbol{M}}\left(\boldsymbol{\tau}_{\boldsymbol{B}}=\mathbf{5} \times \mathbf{1 0}^{-\mathbf{7}}\right)$ & $\boldsymbol{T P}_{\boldsymbol{B}}$ & $\boldsymbol{F} \boldsymbol{N}_{\boldsymbol{B}}$ & $\boldsymbol{F} \boldsymbol{P}_{\boldsymbol{B}}$ & Time [s] \\
\hline 0.1 (1 layer) & 413 & 400 & 28,090 & 2172 \\
0.5 (1 layer) & 305 & 500 & 13,317 & 502 \\
2-3 (2 layers) & 135 & 670 & 1505 & 12 \\
\hline
\end{tabular}




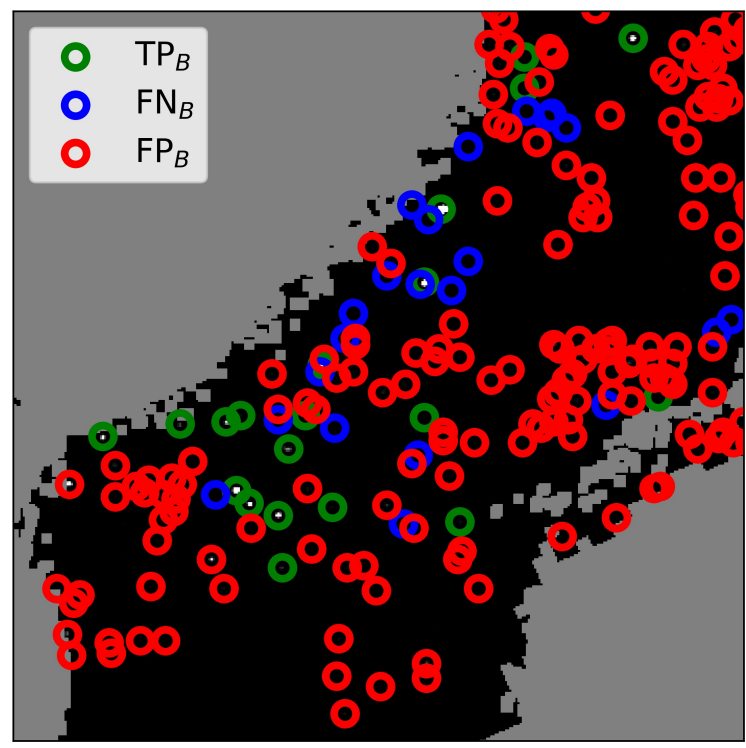

Figure 8. Detected blobs in comparison to manually detected icebergs for $\tau_{B}=5 \times 10^{-7}$ and $S_{M}=0.1$ over FJL on 4 April 2017. Grey areas are land. Blobs are classified according to Table 3.

Table 8. Blob detection results for each of the four images using $\tau_{B}=5 \times 10^{-7}$ and $S_{M}=0.1$. The total number of icebergs identified in the Sentinel-2 images (outside the landmasks) is $T P_{B}+F N_{B}$. The last column indicates the computation time in seconds. For icebergs in rough fast ice, the number of blobs and hence the computation time is considerably larger than for icebergs in smooth fast ice.

\begin{tabular}{cccccc}
\hline $\begin{array}{c}\text { Image Data } \\
\text { Date (Year-Month-Day) Time }\end{array}$ & $\boldsymbol{T P}_{\boldsymbol{B}}$ & $\boldsymbol{F N _ { \boldsymbol { B } }}$ & $\boldsymbol{F \boldsymbol { P } _ { \boldsymbol { B } }}$ & $\begin{array}{c}\text { Part of Image } \\
\text { [No of Rows, } \\
\text { No of Columns] }\end{array}$ & Time [s] \\
\hline 2017-04-04 11:46:41 & 413 & 400 & 28,090 & $3000 \times 3000$ & 2172 \\
2017-04-07 11:56:40 & 370 & 310 & 14,307 & $2500 \times 2000$ & 563 \\
2017-04-10 13:47:25 & 65 & 47 & 148,299 & $4000 \times 4000$ & 54,880 \\
2017-04-19 14:17:39 & 94 & 86 & 99,282 & $3000 \times 4300$ & 26,322 \\
\hline
\end{tabular}

\subsection{PDF Fitting}

\subsubsection{Choosing $W_{C F A R}$}

To select the preferable window size for the CFAR detector we have calculated the mean absolute difference between each selected PDF and the corresponding histogram for three different window sizes, denoted $W_{C F A R}$ in Table 9. We found that, in general, there was a trend that the larger the window size the more robust are the PDFs. However, with larger window sizes we also increase the computational effort. Therefore, we did not use a window larger than $141 \times 141$ pixels.

Table 9. The mean and standard deviation of the mean absolute difference (in percent) between the PDFs calculated for the window around each blob and the corresponding histogram of the data between $I_{\min }$ and $I_{\max }$. For the test we used a subscene of the Sentinel-1 image from 4 April 2017, covering approximately 9700 blobs. The green row corresponds to the preferable parameters.

\begin{tabular}{cccc}
\hline$W_{\text {CFAR }}$ & Gamma & Generalized Gamma & GEV \\
\hline $61 \times 61$ & $0.21 \pm 0.08$ & $0.19 \pm 0.06$ & $0.19 \pm 0.08$ \\
$101 \times 101$ & $0.19 \pm 0.08$ & $0.16 \pm 0.06$ & $0.18 \pm 0.07$ \\
$141 \times 141$ & $0.19 \pm 0.12$ & $0.15 \pm 0.07$ & $0.17 \pm 0.09$ \\
\hline
\end{tabular}




\subsubsection{Representability and Quality of PDFs}

Table 10 shows the differences (in percent) between a calculated PDF and the corresponding normalized histogram for the three PDFs presented in Section 3.4.2. The differences were calculated both for a subimage of $3000 \times 3000$ pixels and for all windows within the subimage. The window size was $141 \times 141$ pixels. The Generalized Gamma distribution reveals the best fit to the data both with respect to the full image and to the sub-images. The Generalized Gamma PDF is thus used in the following investigations.

Table 10. Mean of relative differences (in percent) between PDF and histogram from $I_{\min }$ to $I_{\max }$ for the subimage from 4 April 2017, and average and standard deviation of the mean differences for all windows of $141 \times 141$ pixels in size, respectively.

\begin{tabular}{cccc}
\hline & Gamma [\%] & Generalized Gamma [\%] & GEV [\%] \\
\hline Full image & 0.28 & 0.17 & 0.19 \\
Sub-images & $0.19 \pm 0.12$ & $0.15 \pm 0.07$ & $0.17 \pm 0.09$ \\
\hline
\end{tabular}

\subsubsection{TIP}

The 'Tail Integrated Probability' (TIP) was calculated using the Generalized Gamma distribution with $W_{C F A R}=141$, and the parameters for the distribution were determined from the background as described in Section 3.4.1. In Figure 9 the TIP values for all detected blobs are presented as functions of the iDPolRAD ratio, including icebergs (true positives) and non-icebergs (false positives). The idea was to separate the icebergs from the non-icebergs by choosing an optimal PFA value, for example as maximum of all TIPs obtained for true positives (assuming that the TIPs for non-icebergs are larger than the ones for icebergs).

From Figure 9, we have to conclude that it is difficult to unambiguously distinguish true and false positives based on the TIP. This is due to the fact that many icebergs cover the same range of iDPolRAD values as the non-icebergs, and many non-icebergs have a high pixel-value relative to their neighborhood and will thus stand out as an object with low TIP. If the PFA is chosen very high many icebergs are detected but the number of false alarms is also high. If the PFA is chosen very low, the number of false alarms is reduced at the cost of loosing many icebergs (see Table 11 below).

For many blobs the blob value is very large $\left(I_{b l o b}>I_{\max }\right)$, resulting in an extremely low TIP value. This happens, for example, if an iceberg with particularly high iDPolRAD value is present in completely smooth and homogeneous fast ice which reveals very low values. In such cases, we set the TIP values equal to 0 , which means that the probability that any pixel value of the background is larger than the corresponding $I_{b l o b}$ is zero. In Figure 9, we plotted only the iDPolRAD values of blobs for which the TIP is larger than $10^{-10}$. Objects with TIP $<10^{-10}$ will be detected in any case since the PFA-values we chose in Table 11 are higher.

Another problem occurs if there is a larger number of objects in the training window (e.g., other icebergs or sea ice deformation structures) for which $\mathrm{I}<I_{\max }$ but which are bright compared to the other background pixels. They influence the tail of the background PDF, and we obtain a larger value of the TIP. 


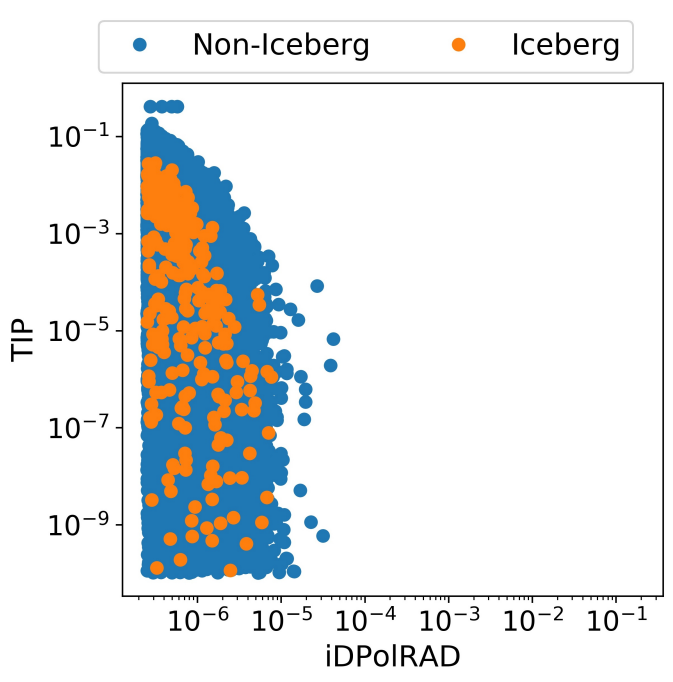

(a)

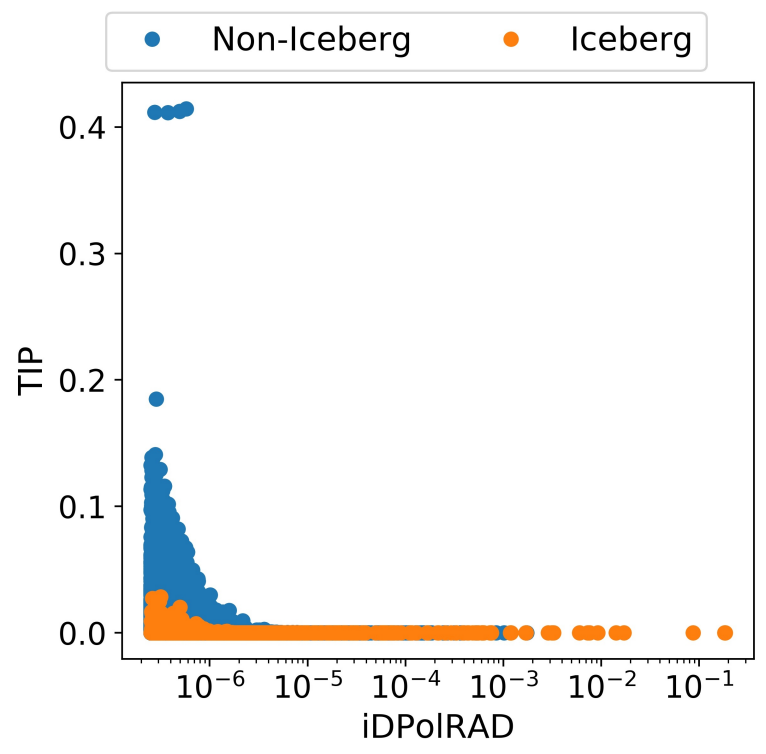

(b)

Figure 9. TIP values for all blob-detected pixels - both icebergs (orange) and non-icebergs (blue) for the Generalized Gamma distribution. These data includes both dates from the area of smooth ice (4 and 7 April 2017). Note that in (a) both axes are in log-scale, while in (b) only the $x$-axis is in logarithmic scale.

\subsection{Confusion Matrix}

Table 11 presents the automatic detection results for a number of different PFAs using the Generalized Gamma PDF with $W_{C F A R}=141$ for the four scenes in Figure 1. Only the blob-detected pixels were included in this detection. This means that we have already lost about $50 \%$ of the icebergs (Table 8). The different PFAs were chosen based on the result in Figure 9; they are chosen at those TIPs where we found abrupt changes in the number of true detections. The final PFA is chosen from the results in Table 11. Naturally a higher PFA results in larger values of $F P_{C F A R}$-coupled with a higher $T P_{C F A R}$. For the selected PFAs the $F P_{C F A R}$ is more than 10 times as large as $T P_{C F A R}$ (Table 11). This means that only every tenth detected pixel (or less) is a real iceberg. If the number of false alarms per unit area cannot be neglected in comparison to the true positives, an unrealistic iceberg areal density is obtained. Table 12 show that approximately $20 \%$ of the icebergs are detected in the smooth fast ice, while less than $10 \%$ are identified in the rough fast ice.

\subsection{Comparing Detection Results with and without Blob Detection}

To check whether the first step of blob detection is useful or not we compare the results of the complete processing chain as shown in Figure 4 with the results of excluding the blob detection and only using the CFAR approach. Because it is a very time consuming task when fitting the Generalized Gamma function to the background values each time, we calculated the threshold of the iDPolRAD value only for every tenth pixel and applied linear interpolation to cover the remaining pixels. In the calculations we used a PFA of $10^{-6}$. The difference in the computation time for the blob detection as first step and the CFAR approach (only applied on blobs) as a subsequent step is large. In our implementation the blob-detector (with $S_{M}=0.1$ and $\tau_{B}=5 \times 10^{-7}$ ) needed about $36 \mathrm{~min}$ for the image 4 April 2017 11:46:41 (see Table 8), and the subsequent CFAR detection approximately $24 \mathrm{~h}$. The same time was needed for fixing the threshold for every 10th pixel, interpolating threshold values between them, and carry out the iceberg detection for every pixel. The long computation times needed for the CFAR detector are mainly caused by fitting and numerically integrating the Generalized Gamma function to the background values. For practical applications, this procedure is not directly applicable. The true detection rate is higher for the pixel-by-pixel CFAR detector (271 true detections) compared to 
including the preceding blob detector (178 true detections). With the two-step approach, 400 icebergs were missed already in the blob-detection step (Table $8, F N_{B}$ ). However, while the pixel-by-pixel detection resulted in 3980 false alarms (Figure 10a), the detector with the preceding blob-detector ended with 2890 false alarms (Figure 10b). We emphasize that the numbers of false alarms (FP) that we provide in Table 9 are not directly related to the PFA, for which we take only into account the high iDPolRAD values in a SAR image. Here the number of false alarms is determined in comparison to the reference data from the optical images. False alarms are bright signals in the radar image that do not correspond to an iceberg position in the optical image. SAR pixels covering icebergs that do not show high values in the SAR image are part of the background PDF but are counted as missed iceberg in comparison to the optical images. The large number of false alarms (FP) and missed icebergs (FN) demonstrates that it is difficult to reliably distinguish icebergs from other bright appearing objects in the SAR image and that not all icebergs are objects with a strong microwave backscattering response.

Table 11. Confusion matrix for three PFA values for the data in each respective scene. Only blob detected pixels are included in the table meaning that for example $F N_{C F A R}$ is relative to $T P_{B}$.

\begin{tabular}{lllll}
\hline PFA & TP $_{\text {CFAR }}$ & $F N_{\text {CFAR }}$ & $F_{\text {CFAR }}$ & $T_{\text {CFAR }}$ \\
\hline $\mathbf{2 0 1 7 - 0 4 - 0 4}$ & & & & \\
\hline $10^{-3}$ & 237 & 176 & 10,182 & 17,908 \\
$10^{-6}$ & 178 & 235 & 2890 & 25,200 \\
$10^{-9}$ & 151 & 262 & 1273 & 26,817 \\
\hline $\mathbf{2 0 1 7 - 0 4 - 0 7}$ & & & & \\
\hline $10^{-3}$ & 184 & 186 & 4565 & 9742 \\
$10^{-6}$ & 121 & 249 & 1685 & 12,622 \\
$10^{-9}$ & 88 & 282 & 906 & 13401 \\
\hline $\mathbf{2 0 1 7 - 0 4 - 1 0}$ & & & & \\
\hline $10^{-3}$ & 21 & 44 & 44,007 & 4292 \\
$10^{-6}$ & 11 & 54 & 15,679 & 132,620 \\
$10^{-9}$ & 10 & 55 & 7941 & 140,358 \\
\hline $\mathbf{2 0 1 7 - 0 4 - 1 9}$ & & & & \\
\hline $10^{-3}$ & 34 & 60 & 26,445 & 72,837 \\
$10^{-6}$ & 15 & 79 & 8590 & 90,692 \\
$10^{-9}$ & 8 & 86 & 4240 & 95,042 \\
\hline
\end{tabular}

Table 12. Relative values (in percent) for each of the scenes in Table 11 , using PFA $=10^{-6}$. Second column gives the proportion of false positives to the total number of detections, while column three and four gives the proportion of true detection and misses to the total number of icebergs, respectively.

\begin{tabular}{cccc}
\hline Date (Year-Month-Day) & $\frac{F P_{C F A R}}{T P_{C F A R}+F P_{C F A R}}[\%]$ & $\frac{T P_{C F A R}}{T P_{C F A R}+F N_{C F A R}+F N_{B}}[\%]$ & $\frac{F N_{C F A R}+F N_{B}}{T P_{C F A R}+F N_{C F A R}+F N_{B}}[\%]$ \\
\hline $2017-04-04$ & 94.2 & 21.9 & 78.1 \\
$2017-04-07$ & 93.3 & 17.8 & 82.2 \\
$2017-04-10$ & 99.9 & 9.7 & 90.3 \\
$2017-04-19$ & 99.8 & 8.3 & 91.7 \\
\hline
\end{tabular}




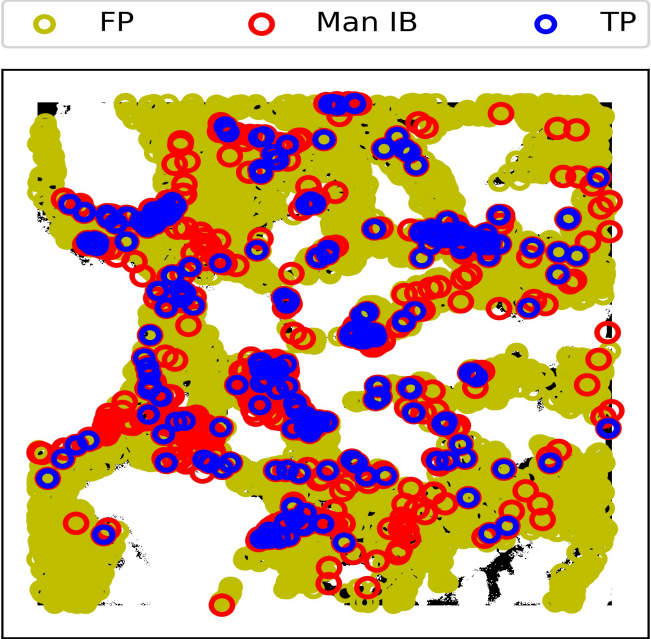

(a)

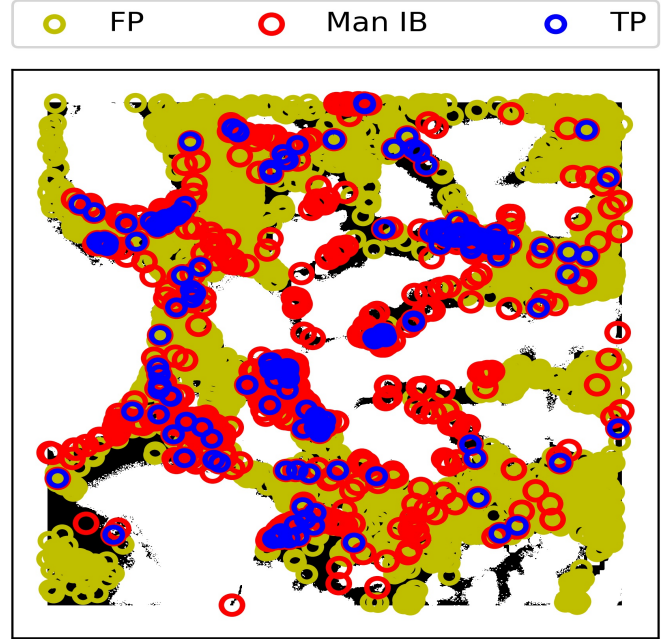

(b)

Figure 10. Detection results using (a) pixel-by-pixel and (b) blob-detection as a first step. Red circles are manually detected icebergs, blue circles are true positive detected icebergs, and yellow circles are false alarms. Landmasks are white. Small bright dots are due to high iDPolRAD values.

\subsection{Testing Algorithm for Different Resolutions}

To check whether a higher resolution-image improves the detection-results we applied the algorithm to a Sentinel-1 IWS image. An area representing smooth sea ice at FJL on 1 April 2017, is used for the comparison. The area contains 101 manually detected icebergs. However, some of the icebergs are removed by the land- and glacier-filter, leaving 68 for the EWS scene and 85 for the IWS scene. The lower number of remaining icebergs for the EWS image is due to a broader smoothing at the border of the landmask which is a result of the coarser spatial resolution. Note that compared to Table 11, a smaller area was covered which included all true detections but less false alarms.

The images were acquired with a temporal separation of $45 \mathrm{~min}$. They were both acquired on descending passes but on different orbits. Therefore, the images differ in their orientation relative to north. The parameters for the blob-detector in the IWS images were selected such that the physical blob-size (in meters) equals the blob-size used for the EWS image. The value of $\tau_{B}$ was increased to better represent the iDPolRAD-values obtained for the IWS mode.

The results in Tables 13 and 14 show that a larger portion of the icebergs are detectable in the IWS mode $(38.8 \%)$ relative to the EWS mode $(20.6 \%)$. However, many more false alarms occur in IWS than in EWS mode (87.5\% against $41.6 \%$ of all detections). This is because the higher resolution in the IWS mode emphasizes more details than the EWS mode, which results in both a larger number of icebergs and a larger number of false alarms. The major problem is that also in IWS mode many icebergs cover only 1-3 pixels, which means that their iDPolRAD signature is hence difficult to distinguish from similar signatures of structures in the fast ice. The speckle contribution is larger at IWS mode $(\mathrm{ENL}=4.4)$ compared to EWS $(\mathrm{ENL}=10.7)$. Narrow deformation structures in the fast ice show up more clearly in the SAR image.

Table 13. Blob detection results for two Sentinel-1 images on 1 April 2017, with different modes. The EWS uses $\tau_{B}=5 \times 10^{-7}$ and $S_{M}=0.1$, while IWS uses $\tau_{B}=1 \times 10^{-4}$ and $S_{M}=0.9$. The total number of icebergs identified in the Sentinel-2 images (outside the landmasks) is $T P_{B}+F N_{B}$.

\begin{tabular}{lccc}
\hline Acquisition Mode & $\boldsymbol{T P}_{\boldsymbol{B}}$ & $\boldsymbol{F N _ { \boldsymbol { B } }}$ & $\boldsymbol{F P}_{\boldsymbol{B}}$ \\
\hline Sentinel-1 EWS & 29 & 39 & 221 \\
Sentinel-1 IWS & 34 & 51 & 426 \\
\hline
\end{tabular}


Table 14. Relative values for CFAR-detection (PFA $=10^{-6}$ ) using the blob-detected pixels in Table 13. Second column gives the proportion of false positives to the total number of detections, while column three and four gives the proportion of true detection and misses to the total number of icebergs, respectively.

\begin{tabular}{lccc}
\hline Acquisition Mode & $\frac{F P_{C F A R}}{T_{C F A R}+F P_{C F A R}}[\%]$ & $\frac{T P_{C F A R}}{T P_{C F A R}+F N_{C F A R}+F N_{B}}[\%]$ & $\frac{F N_{C F A R}+F N_{B}}{T P_{C F A R}+F N_{C F A R}+F N_{B}}[\%]$ \\
\hline Sentinel-1 EWS & 41.6 & 20.6 & 79.4 \\
Sentinel-1 IWS & 87.5 & 38.8 & 61.2 \\
\hline
\end{tabular}

\section{Discussion}

We focused on a SAR image product with a relatively coarse spatial resolution (but wider coverage) since such products are preferably used in operational monitoring of sea ice conditions. As mentioned in the introduction and in Section 4.2, many small icebergs (more than 50 percent of all identified ones) were located very close to the coast. They were partly hidden by the landmask (see Figure 3). In many cases, the training window was overlapping the land so that the corresponding icebergs could not be included in the analysis. The number of the remaining icebergs was nevertheless large enough to yield statistically meaningful results. In practice the detection of icebergs located very close to land is important. This can be achieved by using adaptive training windows in which the test window is not located in the center but close to one margin.

As our results show, many icebergs cannot be recognized in the Sentinel-1 SAR EWS images, even if we make use of the contrast enhancement between iceberg and background inherent in the iDPolRAD filter. When using IWS mode images with a spatial resolution of $20 \mathrm{~m}$ (instead of EWS mode with $90 \mathrm{~m}$ ), more icebergs are detected, and the number of missed icebergs after CFAR-detection is low. However, we also found that the number of false alarms increases when using the IWS mode. Even though an iceberg may be large enough to cover more than one pixel in the Sentinel-1 images it may not be visible due to its shape and due to environmental conditions. The visibility depends on the specific backscattering of the iceberg which is determined by the scattering contributions from its surface and volume and by multiple scattering from interactions between surface, bottom, sidewalls, and volume (e.g., [24]). For example, tabular bergs may not be visible under melting conditions because they are flat and therefore reflect the incident radiation away from the direction to the satellite. We found that many non-iceberg pixels yield the same magnitude of the iDPolRAD-value as iceberg-pixels, causing a large number of false alarms. In particular rough sea ice (as for the test site at Nord-Austlandet) reveals radar scattering characteristics similar to icebergs [24].

To deepen the understanding of the output obtained from the iDPolRAD filter, we investigated the backscattering characteristics (from the backscattering coefficients $\sigma_{H H}^{0}$ and $\sigma_{H V}^{0}$ derived from the EWS images). Figure 11 presents the values of icebergs and non-icebergs, both for iDPolRAD and for $\sigma_{H H}^{0}$ and $\sigma_{H V}^{0}$. The rightmost plot of Figure 11 reveals that backscattering coefficients of icebergs and non-icebergs cover the same range of values, and the gain in using the iDPolRAD approach becomes clear. However, only at larger magnitudes of $\sigma_{H H}^{0}$ and $\sigma_{H V}^{0}$ the difference between iDPolRAD values of icebergs and non-icebergs is significant (Figure 11a). This effect is more pronounced at HV-polarization. For icebergs with lower backscattering intensities, however, the iDPolRAD values of icebergs and non-icebergs are similar. This can be interpreted such that not all icebergs are characterized by scattering mechanisms causing strong depolarization. We expect this, e.g., for icebergs with a wet surface or with very low volume scattering contributions due to the lack of strong scatterers in the ice. For the rough fast ice the difference in iDPolRAD values between icebergs and non-icebergs are not as large as for smooth fast ice (Figure 11b). This can be explained by the low backscattering magnitudes of icebergs at HV-polarization in the NA-scenes. 

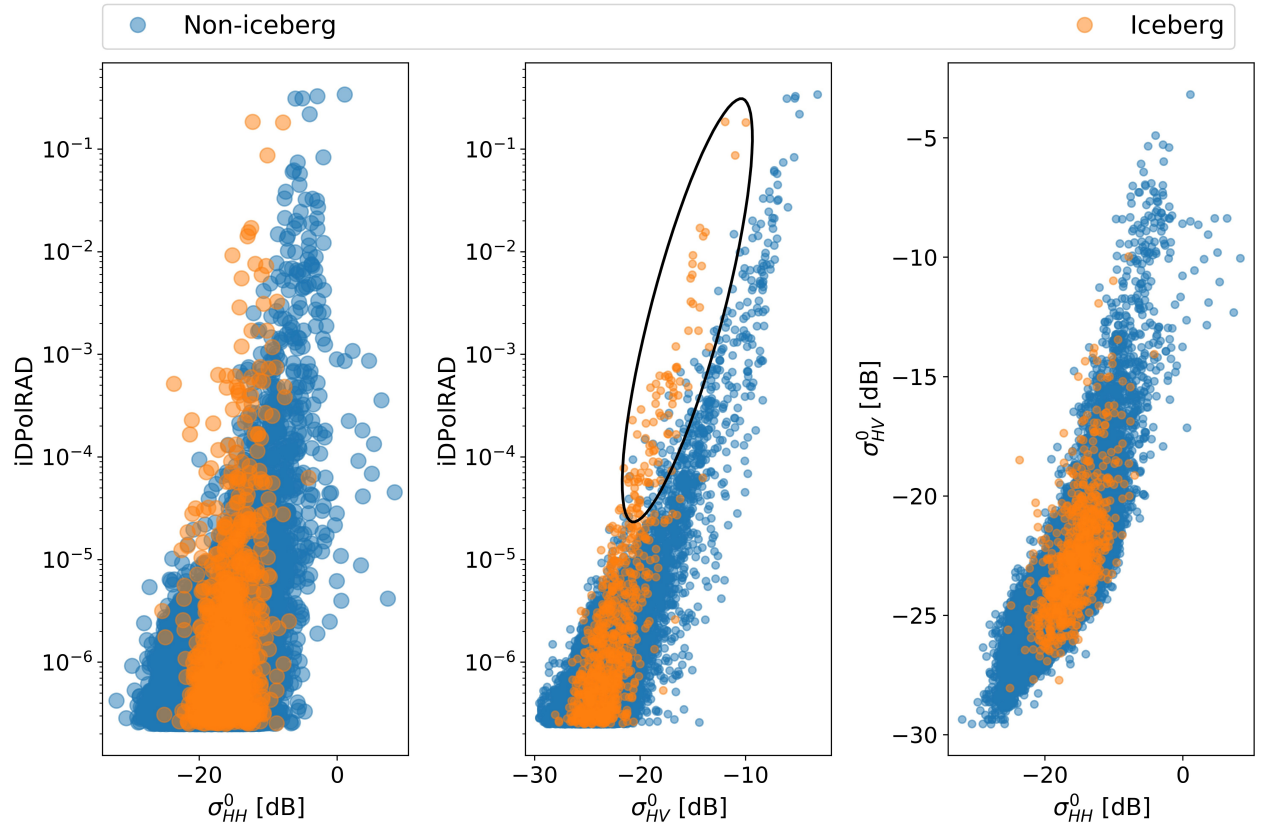

(a)
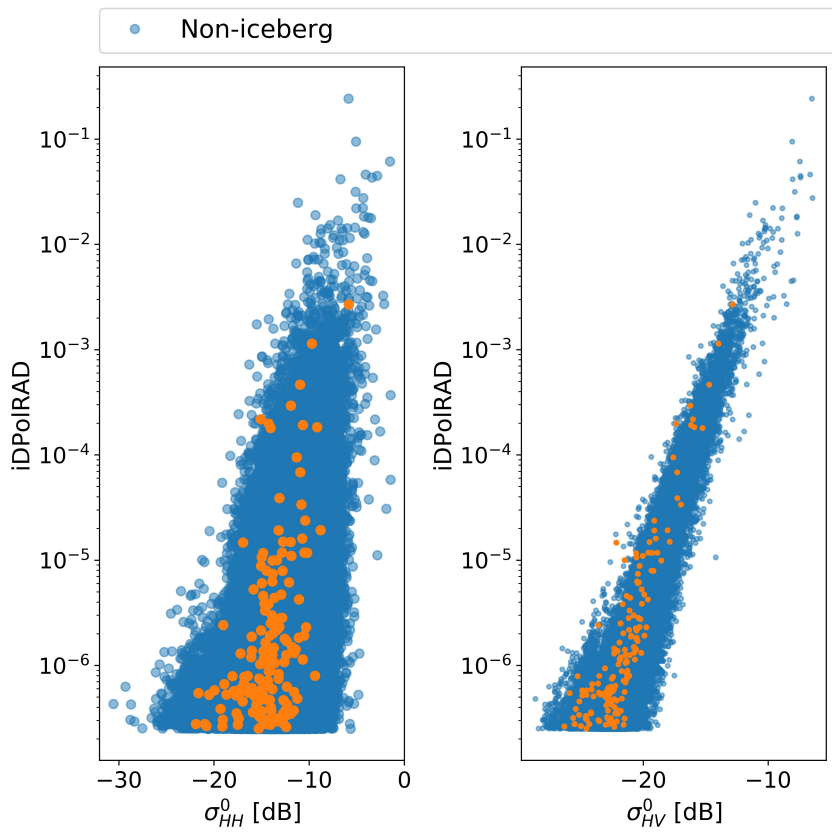

- Iceberg

(b)

Figure 11. Relationships between backscattering coefficients at HH- and HV-polarization and the iDPolRAD for blob-detected icebergs and non-icebergs. Data are from (a) FJL, representing smooth fast ice, and (b) NA, representing rough fast ice.

As mentioned in Section 3.2, the output of the iDPolRAD filter may also be negative. To check how often this situation occurs we plotted $\sigma_{H V}^{0}$ of manually identified icebergs (test window) vs. $\sigma_{H V}^{0}$ of the background (training window). Negative iDPolRAD values occur if the background $\sigma_{H V}^{0}$ is larger than the iceberg $\sigma_{H V}^{0}$ (see Equation (1)). Figure 12 demonstrates that such cases occur frequently in practise, i.e., icebergs that are only weakly scattering objects are embedded in a background containing one or several objects with a strong scattering response (values below orange line). Negative values of iDPolRAD are not considered in the blob detection, hence all negatives are lost in our processing chain 
shown in Figure 4. If the CFAR detector is applied without preceding blob detection, the threshold determined from the PDF of the background can be $<0$ if the background reveals negative iDPolRAD values. A detection of a dark iceberg is successful if the iDPolRAD-value of the iceberg is negative but larger than the average iDPolRAD-value of the background (hence a "dark" iceberg in a "darker" background). Nevertheless in conclusion this means that a separate detection scheme has to be applied for "dark" icebergs in a bright background. This item was, e.g., investigated in Wesche and Dierking [2] who focused on using single-polarization backscattering coefficients. The major point here is that even the dual-polarization approach, for Sentinel-1 linked with a relatively high NESZ, is not able to detect all icebergs and reduce the number of false alarms significantly. The reason is that microwave scattering patterns from icebergs and background (sea ice, open ocean) obtained at C-band at $\mathrm{HH}$ and HV-polarization still reveal similarities (e.g., icebergs and deformed sea ice) that prevent a robust separation. On the other hand, many icebergs in the Barents Sea are too small relative to the spatial resolution of the operationally used EWS images to include a shape analysis as an additional criterion for iceberg detection. Environmental conditions like variable wind fields over open water and high temperatures causing melting complicate the detection process.

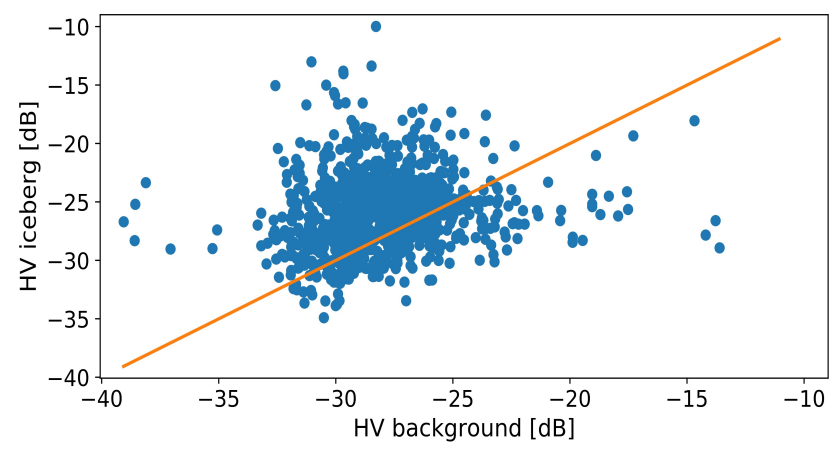

(a)

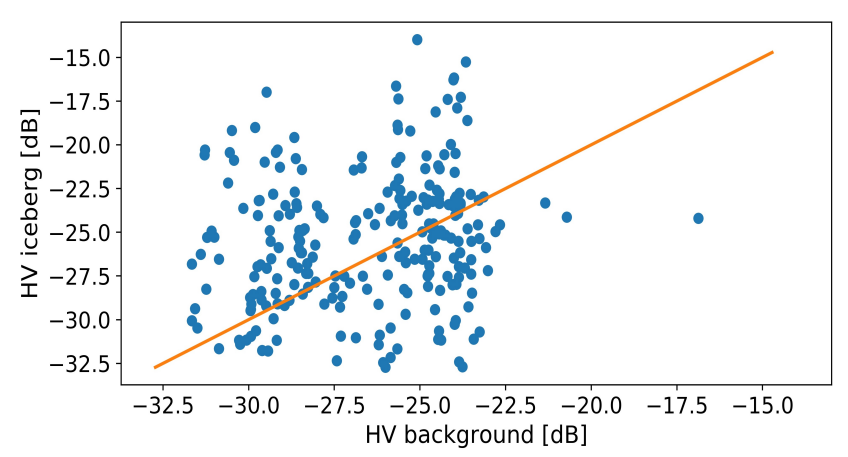

(b)

Figure 12. Backscattering coefficients of icebergs and their corresponding background at HV-polarization. Data are from (a) FJL, representing smooth fast ice, and (b) NA, representing rough fast ice. The orange line indicates where the iceberg value equals the background value.

Finally, we investigated the effect of varying radar incidence angles on the detection performance. From sea ice classification in wide-swath images it is known that changes of the incidence angle have to be considered (e.g., [29]). For all blob detected icebergs and their corresponding background (mean value over the training-window) the iDPolRAD-value and backscattering coefficients at $\mathrm{HH}$ or HV-polarization are retrieved with the corresponding incidence angle. The results are presented in Figure 13. The iDPolRAD-values do not reveal any clear sensitivity to the incidence angle in the investigated range from 28 to $46 \mathrm{deg}$. At a given incidence angle, the backscattering coefficients show large variations both for the icebergs and for the background. Also in this case a clear sensitivity to 
the incidence angle is not recognizable. However, the background backscattering coefficients reveal a slight decrease with increasing incidence angles in particular at smaller values of $\sigma_{H H}^{0}$ and $\sigma_{H V}^{0}$. The latter may arise from smooth fast ice areas (between ridges or other deformation features) for which a decrease of the backscattered intensity as a function of incidence angle is to be expected. For volume scattering from air bubbles in the fast ice volume and for ice ridges, the sensitivity to the incidence angle is in general only weak for the incidence angle range shown here [30].
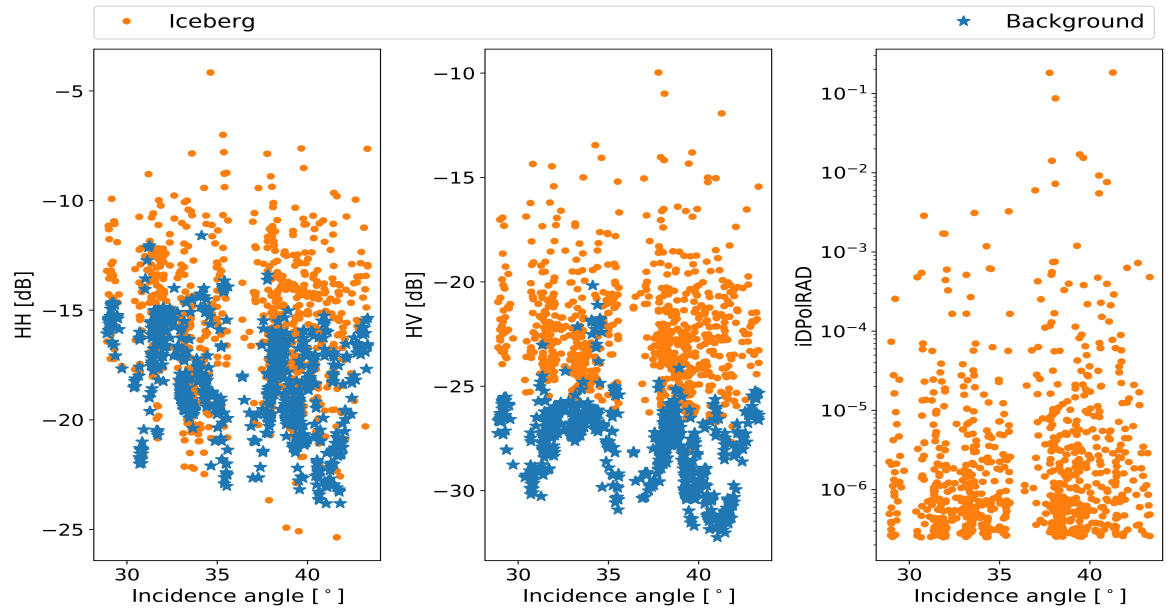

(a)
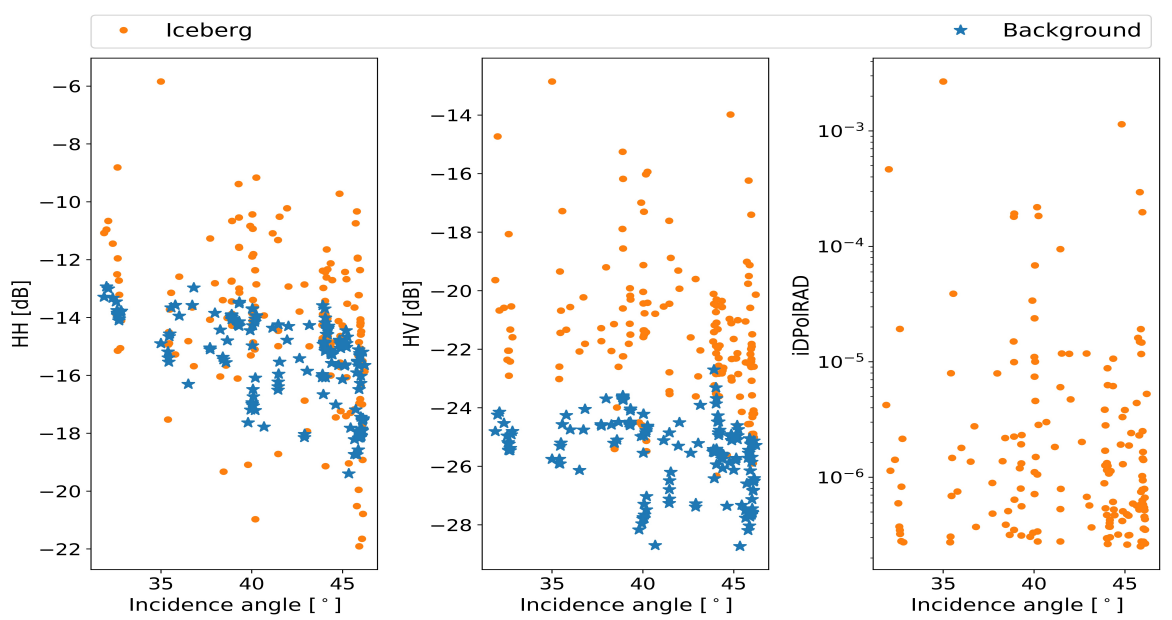

(b)

Figure 13. iDPolRAD values and backscattering coefficients at HH-polarization and HV-polarization vs incidence angle for blob detected pixels. Orange dots are true icebergs while blue stars are the corresponding background values. The images represents (a) smooth ice, and (b) rough ice, each containing both images for each test site. Note that the iDPolRAD values are a function of the background and we therefore only represent icebergs compared to the incidence angles. Note also that the iDPolRAD values are in log-scale.

\section{Conclusions}

In this paper we have investigated the possibility to detect small icebergs between a few meters and $100 \mathrm{~m}$ in size using Sentinel-1 EWS C-band data acquired over smooth and rough fast ice in the Barents Sea. We validated the detection results in comparison to about 2000 icebergs manually identified in optical images. Our investigations focus on the potential and limitations of detecting icebergs with sizes on the order of the SAR spatial resolution typical for data products employed for 
operational ice charting. Many of the icebergs in the Barents Sea are comparable or smaller in size than the spatial resolution of the Sentinel-1 EWS mode, which is $90 \mathrm{~m}$.

As a starting point we selected the output of the iDPolRAD filter proposed by Marino et al. [12] which compares the cross-polarization intensities of an object and the background and is in many cases characterized by a strongly increased image contrast between icebergs and background (sea ice or open ocean) compared to radar intensities at single polarization. Our results indicate that the output of the iDPolRAD filter is not affected by the incidence angle. To speed-up the detection process we used a blob-detector as a first step to pre-select pixels that potentially could represent icebergs, and applied a CFAR algorithm on each of these pixels for final iceberg detection. We tested three different PDFs for theoretically describing the distributions of iDPolRAD-values and found the Generalized Gamma to provide the best fit. The PDF was then used to select a PFA and calculate the threshold for the CFAR detector. To this end we introduced a concept called TIP (Tail Integrated Probability). We found, however, that the TIP did not provide any extra gain for detection of small icebergs in land fast ice. The number of successful iceberg detections increase if the much more time-consuming pixel-by-pixel CFAR approach is used and the blob detector is omitted, but the number of false alarms is higher as well.

Even with our more sophisticated (but time-consuming) detection scheme, one obtains a relatively low number of true detections and a high number of false alarms and missed icebergs when using the Sentinel-1 EWS images. Also when employing the IWS mode, the number of identified icebergs is still low and the rate of false alarms high. Many icebergs that we could identify in the optical images did not cause a strong radar response in both SAR modes. In an investigation of the backscattering characteristics at $\mathrm{HH}$ - and HV-polarization we found that (a) not all icebergs (even larger ones) are strong scatterers at HV-polarization, and (b) scattering intensities at HV-polarization from structures present on fast ice (such as ridges, hummocks, cracks) can be similar to those from icebergs. Icebergs with relatively low HV-polarized scattering responses are not detected, and strong-scattering ice structures cause false alarms.

\section{Data Availability}

Python scripts for detection and analyzing the data can be achieved by contacting the first author. SAR and MSI images are freely available at https://scihub.copernicus.eu/.

Author Contributions: Conceptualization, I.H.S. and W.D.; Methodology, I.H.S., A.K., and W.D.; Software, I.H.S. and A.K.; Validation, I.H.S., W.D., and A.K.; Formal Analysis, I.H.S.; Investigation, I.H.S., A.K., and W.D.; Resources, I.H.S.; Data Curation, I.H.S.; Writing-Original Draft Preparation, I.H.S., W.D., and A.M.; Writing-Review \& Editing, I.H.S., W.D., A.K., and A.M.; Visualization, I.H.S.; Supervision, A.K. and W.D.

Funding: This research was funded by CIRFA partners and the Research Council of Norway (RCN) (grant number 237906).

Acknowledgments: I.H.S. and W.D. would like to thank Torbjørn Eltoft, University of Tromsø, for stimulating discussions and guidance during the work of this project.

Conflicts of Interest: The authors declare no conflict of interest. 


\section{Abbreviations}

The following abbreviations are used in this manuscript:

$\begin{array}{ll}\text { CFAR } & \text { Constant False Alarm Rate } \\ \text { FJL } & \text { Franz Josef Land } \\ \text { ENL } & \text { Equivalent Number of Looks } \\ \text { EWS } & \text { Extra Wide Swath } \\ \text { GEE } & \text { Google Earth Engine } \\ \text { GEV } & \text { Generalized Extreme Value } \\ \text { iDPolRAD } & \text { intensity Dual-Polarization Ratio Anomaly Detector } \\ \text { IWS } & \text { Interferometric Wide Swath } \\ \text { MSI } & \text { Multi Spectral Imager } \\ \text { NA } & \text { Nord-Austlandet } \\ \text { NESZ } & \text { Noise Equivalent Sigma Zero } \\ \text { PDF } & \text { Probability Density Function } \\ \text { PFA } & \text { Probability of False Alarm } \\ \text { SAR } & \text { Synthetic Aperture Radar } \\ \text { TIP } & \text { Tail Integrated Probability }\end{array}$

\section{References}

1. Jackson, C.R.; Apel, J.R. Synthetic Aperture Radar: Marine User's Manual; US Department of Commerce, National Oceanic and Atmospheric Administration, National Environmental Satellite, Data, and Information Service (NESDIS), Office of Research and Applications: Washington, DC, USA, 2004; p. 411.

2. Wesche, C.; Dierking, W. Iceberg signatures and detection in SAR images in two test regions of the Weddell Sea, Antarctica. J. Glaciol. 2012, 58, 325-339. [CrossRef]

3. Silva, T.A.; Bigg, G.R. Computer-based identification and tracking of Antarctic icebergs in SAR images. Remote Sens. Environ. 2005, 94, 287-297. [CrossRef]

4. Power, D.; Youden, J.; Lane, K.; Randell, C.; Flett, D. Iceberg detection capabilities of RADARSAT synthetic aperture radar. Can. J. Remote Sens. 2001, 27, 476-486. [CrossRef]

5. Williams, R.; Rees, W.; Young, N. A technique for the identification and analysis of icebergs in synthetic aperture radar images of Antarctica. Int. J. Remote Sens. 1999, 20, 3183-3199. [CrossRef]

6. Willis, C.; Macklin, J.; Partington, K.; Teleki, K.; Rees, W.; Williams, R. Iceberg detection using ERS-1 synthetic aperture radar. Int. J. Remote Sens. 1996, 17, 1777-1795. [CrossRef]

7. Norwegian Petroleum Directorate. NPD FactMaps Standard. Available online: http://gis.npd.no/ Factmaps/html_21/ (accessed on 6 August 2018).

8. Arctic Council. Arctic Marine Shipping Assessment 2009 Report; Arctic Council: Tromsø, Norway, 2009.

9. Keghouche, I.; Counillon, F.; Bertino, L. Modeling dynamics and thermodynamics of icebergs in the Barents Sea from 1987 to 2005. J. Geophys. Res. Ocean. 2010, 115. [CrossRef]

10. Sandven, S.; Babiker, M.; Kloster, K. Iceberg observations in the Barents Sea by radar and optical satellite images. In Proceedings of the ENVISAT Symposium, Montreux, Switzerland, 23-27 April 2007.

11. National Snow and Ice Data Center. Cryosphere Glossary: Fast Ice. Available online: https://nsidc.org/ cryosphere/glossary/term/fast-ice (accessed on 24 July 2018).

12. Marino, A.; Dierking, W.; Wesche, C. A Depolarization Ratio Anomaly Detector to identify icebergs in sea ice using dual-polarization SAR images. IEEE Trans. Geosci. Remote Sens. 2016, 54, 5602-5615. [CrossRef]

13. Gill, R.S. Operational Detection of Sea Ice Edges and Icebergs Using SAR. Can. J. Remote Sens. 2001, 27, 411-432. [CrossRef]

14. Frost, A.; Ressel, R.; Lehner, S. Automated Iceberg Detection Using High-Resolution X-Band SAR Images. Can. J. Remote Sens. 2016, 42, 354-366. [CrossRef]

15. Collecte Localisation Satellites (CLS). Sentinel-1 Product Definition; Report 2.7; European Space Agency: Frascati, Italy, 2016.

16. European Space Agency. User Guide-Sentinel SAR_Level-1 Ground Range Detected. Available online: https:/ / sentinel.esa.int/web/sentinel/user-guides/sentinel-1-sar/resolutions/level-1-ground-rangedetected (accessed on 11 October 2018). 
17. Korosov, A.; Hansen, M.; Dagestad, K.F.; Yamakawa, A.; Vines, A.; Riechert, M. Nansat: A scientist-orientated python package for geospatial data processing. J. Open Res. Softw. 2016, 4. [CrossRef]

18. Miranda, N.; Meadows, P. Radiometric Calibration of S-1 Level-1 Products Generated by the S-1 IPF. 2015 Available online: https:/ /Sentinel.Esa.Int/Doc./247904/685163/S1-Radiom.-Calibration-V1.0.Pdf (accessed on 10 October 2018).

19. Carroll, M.L.; Townshend, J.R.; DiMiceli, C.M.; Noojipady, P.; Sohlberg, R.A. A new global raster water mask at $250 \mathrm{~m}$ resolution. Int. J. Digit. Earth 2009, 2, 291-308. [CrossRef]

20. Korosov, A.A.; Rampal, P. A combination of feature tracking and pattern matching with optimal parametrization for sea ice drift retrieval from SAR data. Remote Sens. 2017, 9, 258. [CrossRef]

21. Gorelick, N.; Hancher, M.; Dixon, M.; Ilyushchenko, S.; Thau, D.; Moore, R. Google Earth Engine: Planetary-scale geospatial analysis for everyone. Remote Sens. Environ. 2017. [CrossRef]

22. Nunziata, F.; Migliaccio, M.; Brown, C.E. Reflection symmetry for polarimetric observation of man-made metallic targets at sea. IEEE J. Ocean. Eng. 2012, 37, 384-394. [CrossRef]

23. Marino, A. A notch filter for ship detection with polarimetric SAR data. IEEE J. Sel. Top. Appl. Earth Obs. Remote Sens. 2013, 6, 1219-1232. [CrossRef]

24. Dierking, W.; Wesche, C. C-Band Radar Polarimetry-Useful for Detection of Icebergs in Sea Ice. IEEE Trans. Geosci. Remote Sens. 2014, 52, 25-37. [CrossRef]

25. Scikit-Image. Blob Detection. Available online: http://scikit-image.org/docs/dev/auto_examples/ features_detection/plot_blob.html (accessed on 24 July 2018).

26. Lindeberg, T. Feature detection with automatic scale selection. Int. J. Comput. Vis. 1998, 30, 79-116. [CrossRef]

27. SciPy. Statistical Functions. Available online: https://docs.scipy.org/doc/scipy/reference/stats.html (accessed on 25 July 2018).

28. Engineering Statistics Handbook. Kolmogorov-Smirnoc Goodness-of-Fit Test. Available online: https: / / www.itl.nist.gov/div898/handbook/eda/section3/eda35g.htm (accessed on 25 July 2018).

29. Dierking, W. Mapping of Different Sea Ice Regimes Using Images From Sentinel-1 and ALOS Synthetic Aperture Radar. IEEE Trans. Geosci. Remote Sens. 2010, 48, 1045-1058. [CrossRef]

30. Dierking, W. Technical Assistance for the Deployment of Airborne SAR and Geophysical Measurements during the ICESAR 2007; Final Report_Part 2: Sea Ice; ESA-ESTEC: Noordwijk, The Netherlands, 2008.

(C) 2019 by the authors. Licensee MDPI, Basel, Switzerland. This article is an open access article distributed under the terms and conditions of the Creative Commons Attribution (CC BY) license (http:/ / creativecommons.org/licenses/by/4.0/). 